\title{
EXTREME EIGENVALUES OF TOEPLITZ FORMS AND APPLICATIONS TO ELLIPTIC DIFFERENCE EQUATIONS $\left({ }^{(}\right)$
}

\author{
BY \\ SEYMOUR V. PARTER
}

1. Introduction. Because of their many applications, primarily in the theory of probability, there has been a renewed interest in the theory of Toeplitz forms (see [6]). Of particular interest has been the work of Szegö on the distribution of the eigenvalues of finite sections of Toeplitz forms. More recently Kac, Murdoch and Szegö [10] obtained estimates on the asymptotic behaviour of the extreme eigenvalues of certain of these finite sections. Later, Widom [19] re-obtained these results and extended them, under suitable restrictions.

At the same time, recent interest in iterative methods of solving elliptic difference equations has been accompanied by the development of techniques for estimating the extreme eigenvalues of certain "block" matrices. For example, there are techniques using the theory of non-negative matrices (see $[17 ; 18])$, techniques using the classical theory of the direct product of two matrices (see $[7 ; 11]$ ) and the techniques (usually ascribed to Frankel [5], cf. $[1 ; 2])$ of separation of variables.

In a recent work [14] we studied a very special class of block matrices and obtained some partial results. These enabled us to obtain estimates on the rates of convergence of the "two-line" iterative methods of the Laplace and biharmonic difference equations in rectangular domains $\left({ }^{2}\right)$. In the case of Laplace's equation we obtained an exact asymptotic result. However, in the case of the biharmonic equation we obtained only a "one-sided" estimate.

The purpose of this report is two-fold. In $\$ \S 2,3$, and 4 we extend the results of $\mathrm{Kac}$, Murdoch and Szegö, and Widom. We will make very strong use of Widom's results and technique. In $\$ 5$ we discuss the application of the preceding results to the general problem of the extreme eigenvalues of "block" Toeplitz matrices. These include the matrices of elliptic difference equations

Presented to the Society, January 26, 1961; received by the editors October 18, 1960.

(1) Some of these results were obtained while the author was at the Brookhaven National Laboratory, summer 1959.

(2) The "two-line" iterative methods for the Laplace and biharmonic difference equations were studied by R. S. Varga [18] at the same time. His approach is totally different from the one we investigated in [14]. His approach to the solution of the iteration equations is more general and probably preferable. Varga also estimated the rate of convergence in the Laplace case using the theory of non-negative matrices. That theory does not apply to the biharmonic case. 
(of any order) with constant coefficients in rectangular domains. In fact, every Hermitian difference equation (i.e., one which may be represented by a Hermitian matrix) with constant coefficients on a rectangular domain corresponds to a special block Toeplitz matrix, a "two-dimensional" Toeplitz matrix (see \$9), and conversely.

Finally, we apply this method in several examples. In particular, we return to the problem studied in [14] and give a complete discussion. With these results we are able to obtain another estimate for the rate of convergence of the two-line methods for the biharmonic difference equation. Loosely speaking, while the convergence may be faster than indicated in [14], it is not much faster.

I should like to take this opportunity to thank Professor M. Rosenblatt who suggested that the theory of the Toeplitz forms should be applicable to these problems.

Also I want to thank my friends at Indiana University who discussed this work with me. My special thanks goes to Mr. Ernest Davidson.

2. Preliminary results. Let $f(\theta)$ be a real Lebesgue integrable function defined in $[-\pi, \pi]$. Let $\left\{C_{j}\right\}$ be the Fourier coefficients of $f(\theta)$, i.e.

$$
f(\theta) \sim \sum_{-\infty}^{\infty} C_{j} e^{i j \theta}
$$

Definition 1. The matrix $T_{n}[f]=\left(C_{s-j}\right) s, j=0,1,2, \cdots, n$ is called the $n$th finite section of the Toeplitz matrix $\left(C_{s-j}\right)$ associated with the function $f(\theta)$. We frequently will write $T_{n}$ instead of $T_{n}[f]$.

We will be concerned with functions $f(\theta)$ satisfying

Condition A. Let $f(\theta)$ be real, continuous and periodic with period $2 \pi$. Let $\min f(\theta)=f(0)=m$ and let $\theta=0$ be the only value of $\theta(\bmod 2 \pi)$ for which this minimum is attained.

Condition $\mathrm{A}(\alpha)$. Let $f(\theta)$ be a function satisfying Condition A. Moreover, let $f(\theta)$ have continuous derivatives of order $2 \alpha$ in some neighborhood of $\theta=0$. Finally let $f^{(2 \alpha)}(0)=\sigma^{2}>0$ be the first nonvanishing derivative of $f(\theta)$ at $\theta=0$.

The assumption that the minimum of $f(\theta)$ is achieved at $\theta=0$ is unimportant; the point 0 may be replaced by any $\theta_{0}$. Since the Toeplitz matrix corresponding to any translate $\left[f\left(\theta+\theta_{0}\right)\right]$ of $f(\theta)$ has exactly the same eigenvalues as $f(\theta)$, it is easy to modify the results so that they hold in the more general case. Moreover, there is no loss in generality in assuming $m=0$.

In $\$ 4$ we turn to the case of functions $f(\theta)$ satisfying

Condition B. Let $f(\theta)$ be a real, continuous even function and periodic with period $2 \pi$. Let $\min f(\theta)=f\left( \pm \theta_{0}\right)=m$ and let $\pm \theta_{0} \neq 0$ be the only values of $\theta(\bmod 2 \pi)$ for which this minimum is attained.

Condition $\mathrm{B}(\alpha)$. Let $f(\theta)$ be a function satisfying Condition B. Moreover, let $f(\theta)$ have continuous derivatives of order $2 \alpha$ in some neighborhood of 
$\theta=\theta_{0}$ (and a fortiori in some neighborhood of $\theta=-\theta_{0}$ ). Finally let $f^{(2 \alpha)}\left( \pm \theta_{0}\right)$ $=\sigma^{2}>0$ be the first nonvanishing derivative of $f(\theta)$ at $\theta= \pm \theta_{0}$.

Of course all statements concerning the minimum of $f(\theta)$ and corresponding statements about minimal eigenvalues of matrices may be suitably modified to apply to the maximum of $f(\theta)$ and the maximal eigenvalues of matrices.

We first develop a rough, but useful, estimate for $\lambda_{1, n}$, the minimal eigenvalue of $T_{n}$. This estimate is obtained from the well-known characterization of the eigenvalues of a Hermitian matrix as solutions of a variational problem.

Lemma 1 (Weyl-Courant). Let $A$ and $B$ be two Hermitian matrices, the second positive definite. We denote the eigenvalues of $A$ with respect to $B$ by $\lambda_{0}, \lambda_{1}, \cdots, \lambda_{n}$, ordered in nondecreasing order. Then $\lambda_{\nu}, \nu \geqq 1$, can be characterized by the following extremum property

$$
\lambda_{\nu}=\underset{Y_{1}, Y_{2}, \ldots, Y_{n-1}}{\operatorname{Max}} \cdot \operatorname{Min}_{x} \frac{(x, A x)}{(x, B x)},
$$

where $Y_{1}, Y_{2}, \cdots, Y_{\nu-1}$ is an arbitrary set of $\nu-1$ vectors and $x$ runs over all vectors satisfying

$$
\|x\|=1, \quad\left(Y_{k}, x\right)=0, \quad k=1,2, \cdots, v-1 .
$$

Furthermore, for $\nu=0$ we have

$$
\lambda_{0}=\operatorname{Min}_{x} \frac{(x, A x)}{(x, B x)}
$$

where $x$ runs over all nonzero vectors.

Proof. See [3].

It follows from this lemma that if we have two Hermitian matrices $T$ and $U$ such that $(T x, x) \leqq(U x, x)$ for all vectors $x$, then each eigenvalue of $T$ is less than or equal to the corresponding eigenvalue of $U$. In the case of Toeplitz matrices, we see that if we denote the eigenvalues of $T_{n}[f]$ by $\lambda_{r, n}$ and denote the eigenvalues of $T_{n}[g]$ by $\mu_{\nu, n}$, then $f(\theta) \leqq g(\theta)$ for all $\theta$ implies $\lambda_{\nu, n} \leqq \mu_{\nu, n}$ for all $\nu$ and $n$.

Let $X=\left(x_{j}\right) j=0,1, \cdots, n$ be an $(n+1)$ dimensional vector. Let $\phi(\theta)$ $=\sum_{j=0}^{n} x_{j} e^{-i j \theta}$. A direct computation together with Lemma 1 gives

Lemma 2.

$$
\begin{aligned}
\left(X, T_{n} X\right) & =\frac{1}{2 \pi} \int_{-\pi}^{\pi} f(\theta)|\phi|^{2} d \theta \\
\lambda_{1, n} & =\operatorname{Min}_{\phi} \frac{1}{2 \pi} \int_{-\pi}^{\pi} f(\theta)|\phi|^{2} d \theta
\end{aligned}
$$

where 


$$
\phi(\theta)=\sum_{j=u}^{n} C_{j} e^{-i j \theta}
$$

and

$$
\sum_{j=0}^{n}\left|C_{j}\right|^{2}=\frac{1}{2 \pi} \int_{-\pi}^{\pi}|\phi|^{2} d \theta=1 .
$$

LEMMA 3. Let $f(\theta)$ satisfy Conditions $\mathrm{A}$ and $\mathrm{A}(\alpha)$, but otherwise be an arbitrary function.

Then there is a constant $M>0$ such that

$$
\lambda_{1, n} \geqq m+M\left(\frac{1}{n}\right)^{2 \alpha}
$$

Proof. Let $X=\left(x_{j}\right) j=0,1, \cdots, n+1$ be an eigenvector associated with $\lambda_{1, n}$ satisfying $\sum\left|x_{j}\right|^{2}=1$. Let $\phi(\theta)=\sum x_{j} e^{-i j \theta}$. Then by (2.2a) and (2.2b)

$$
\lambda_{1, n}=\frac{1}{2 \pi} \int_{-\pi}^{\pi} f(\theta)|\phi|^{2} d \theta \text {. }
$$

Since $f(\theta)$ satisfies Conditions $\mathrm{A}$ and $\mathrm{A}(\alpha)$, we have, for $n$ sufficiently large

$$
f(\theta) \geqq m+A \theta^{2 \alpha}, \quad-\frac{1}{n} \leqq \theta \leqq \frac{1}{n},
$$

and

$$
f(\theta) \geqq m+A\left(\frac{1}{n}\right)^{2 \alpha}, \quad \frac{1}{n} \leqq|\theta| \leqq \pi ;
$$

where

$$
A=\frac{1}{2} \frac{1}{(2 \alpha) !} \sigma^{2}
$$

Hence

$$
\lambda_{1, n} \geqq m+\frac{A}{2 \pi}\left\{\int_{-n^{-1}}^{n^{-1}} \theta^{2 \alpha}|\phi|^{2} d \theta+\left(\frac{1}{n}\right)^{2 \alpha} \int_{n^{-1} \leq|\theta| \leq \pi}|\phi|^{2} d \theta\right\} .
$$

This gives

$$
\lambda_{1, n} \geqq m+A\left(\frac{1}{n}\right)^{2 \alpha}-\frac{A}{2 \pi} \int_{-n^{-1}}^{n^{-1}}\left[\left(\frac{1}{n}\right)^{2 \alpha}-\theta^{2 \alpha}\right] \cdot|\phi|^{2} d \theta .
$$

Consider the last term. By Schwarz's inequality

$$
|\phi(\theta)|^{2} \leqq n+1<3 n \text {, }
$$

for all $\theta$. 
Hence

$$
\lambda_{1, n} \geqq m+A\left[1-\frac{6}{2 \pi}\left(\frac{2 \alpha}{2 \alpha+1}\right)\right]\left(\frac{1}{n}\right)^{2 \alpha}
$$

and our result follows since $6<2 \pi$.

We now turn to the reverse inequality.

Lemma 4. Let $f(\theta)$ satisfy Condition $\mathrm{A}$ and $\mathrm{A}(\alpha)$. There exists a constant $M_{0}>0$ such that

$$
\lambda_{1, n} \leqq m+M_{0}\left(\frac{1}{n}\right)^{2 \alpha} .
$$

Proof. Let $s=2(\alpha+1)$ and $r=[n / s]$, i.e. $r$ is the largest integer in $n / s$. Then

$$
n-s \leqq r s \leqq n .
$$

Consider the function

$$
g(\theta)=\left[\frac{\sin (r+1 / 2) \theta}{\sin \theta / 2}\right]^{*} \geqq 0 .
$$

It is easy to verify that $g(\theta)$ may be written as

$$
\sum_{-r s}^{r s} C_{j} e^{i j \theta}
$$

and hence $g(\theta)=\left|\phi_{0}\right|^{2}$ for some function,

$$
\phi_{0}(\theta)=\sum_{j=0}^{r s} d_{j} e^{-i j \theta} .
$$

It is also easy to verify that

$$
\frac{1}{2 \pi} \int_{-\pi}^{\pi} g(\theta) d \theta=C_{*} \cdot(2 r+1)^{\circ-1}
$$

where $C_{s}$ can be bounded from above and below by constants independent of $r$. We take $C_{s}^{-1}(2 r+1)^{-(8-1)} \cdot g(\theta)=|\phi|^{2}$ as our "test" function in (2.1b). Lemma 1 gives

$$
\lambda_{1, n} \leqq \frac{1}{2 \pi} \int_{-\pi}^{\pi} f(\theta) \cdot|\phi|^{2} d \theta .
$$

Again, since $f(\theta)$ satisfies Conditions $\mathrm{A}$ and $\mathrm{A}(\alpha)$ there is an $\eta>0$ so that

$$
f(\theta) \leqq m+A \theta^{2 \alpha} \text {, }
$$$$
-\eta \leqq \theta \leqq \eta,
$$

where 


$$
A=2 \frac{1}{(2 \alpha) !} \sigma^{2}
$$

Also, $f(\theta)$ is bounded, i.e., there is a constant $L$ such that

$$
|f(\theta)-m| \leqq L
$$

Let $\eta$ be chosen and fixed, once and for all. Let $n$ be so large that $2 \pi /(2 r+1)$ $<\eta$. Thus

(2.12) $\lambda_{1, n} \leqq m+\frac{A}{\pi} \int_{0}^{2 \pi /(2 r+1)} \theta^{2 \alpha}|\phi|^{2} d \theta+\frac{A}{\pi} \int_{2 \pi /(2 r+1)}^{\eta} \theta^{2 \alpha}|\phi|^{2} d \theta+\frac{L}{\pi} \int_{\eta}^{\pi}|\phi|^{2} d \theta$.

Consider first the last term on the right in (2.12). From (2.11b) and the form of $|\phi|^{2}$ we find

(2.13a) $\frac{L}{\pi} \int_{\eta}^{\pi}|\phi|^{2} d \theta \leqq \frac{L C_{8}^{-1}}{\pi} \frac{1}{\sin \eta / 2}\left[\frac{1}{2 r+1}\right]^{8-1}=L_{1}\left[\frac{1}{2 r+1}\right]^{o-1}$.

Since $\sin \theta \leqq \theta$ and $\sin \theta \geqq 2 \theta / \pi$ for $0 \leqq \theta \leqq \pi / 2$, we find

$$
\begin{aligned}
\frac{A}{\pi} \int_{0}^{2 \pi /(2 r+1)} \theta^{2 \alpha}|\phi|^{2} d \theta & \\
& \leqq \frac{C A}{\pi} C_{:}^{-1}\left[\frac{2 r+1}{2 \alpha+1}\right] \cdot\left[\frac{2 \pi}{2 r+1}\right]^{2 \alpha+1}=L_{2}\left[\frac{1}{2 r+1}\right]^{2 \alpha} .
\end{aligned}
$$

Since $s=2 \alpha+2$ and $\sin \theta \geqq 2 \theta / \pi$ for $0 \leqq \theta \leqq \pi / 2$,

(2.13c) $\frac{A}{\pi} \int_{2 \pi /(2 r+1)}^{\eta} \theta^{2 \alpha}|\phi|^{2} d \theta \leqq \frac{A C^{-1}}{\pi}\left[\frac{1}{2 r+1}\right]^{8-1} \cdot \pi^{*} \int_{2 \pi /(2 r+1)}^{\eta} \frac{d \theta}{\theta^{2}}$.

Hence

$$
\frac{A}{\pi} \int_{2 \pi /(2 r+1)}^{\eta} \theta^{2 \alpha}|\phi|^{2} d \theta \leqq L_{3}\left[\frac{1}{2 r+1}\right]^{2 \alpha} .
$$

Finally, using (2.8), we find

$$
\lambda_{1, n} \leqq m+L_{0}\left[\frac{1}{2 r+1}\right]^{2 \alpha} \leqq m+M_{0}\left[\frac{1}{n}\right]^{2 \alpha} .
$$

We restate Lemmas 3 and 4 as

Theorem 1. Let $f(\theta)$ satisfy Conditions $\mathrm{A}$ and $\mathrm{A}(\alpha)$. Let $\lambda_{1, n}$ be the minimal eigenvalue of $T_{n}[f]$. Then

$$
\lambda_{1, n}=m+O\left(n^{-2 \alpha}\right)
$$


where $O$ cannot be replaced by 0 .

We now turn to the discussion of the technique and method developed by Widom in [19]. From Lemma 2 and the continuity of $f(\theta)$ we see that the eigenvalues $\lambda_{\nu, n}$ may be characterized by certain values $\theta_{v, n}$, where

$$
\lambda_{\nu, n}=f\left(\theta_{\nu, n}\right) .
$$

Theorem 1 tells us that $\theta_{1, n}=O(1 / n)$ and $O$ cannot be replaced by $o$. The method of Widom hinges on the idea of estimating $\theta_{r, n}$ rather than $\lambda_{r, n}$. We will develop these ideas in the general case although we only give a detailed analysis of the case $\alpha=2$.

The case $\alpha=1$ was studied by Kac, Murdoch and Szegö. Widom also studied the case $\alpha=1$ under the restriction that $f(\theta)$ is even and $f(\theta)$ has four continuous derivatives in the neighborhood of $\theta=0$. The result of their works is

Theorem 2. Let $f(\theta)$ satisfy Conditions $\mathrm{A}$ and $\mathrm{A}(\alpha)$ with $\alpha=1$. Let $\lambda_{\nu, n}$ be the eigenvalues of $T_{n}[f]$ arranged in nondecreasing order. Let $\theta_{\nu, n}$ be the corresponding values of $\theta$. Then, as $n \rightarrow \infty$, we have for fixed $\nu, \nu=1,2, \cdots$,

$$
\lambda_{\nu, n}=f\left(\frac{\nu \pi}{n+2}+E_{v, n}\right)=m+\frac{1}{2}\left(\frac{\nu \pi}{n+2}\right)^{2} f^{\prime \prime}(0)+o\left(n^{-2}\right)
$$

where

$$
E_{v, n}=o\left(\frac{1}{n}\right)
$$

That is

$$
\theta_{\nu, n}=\frac{\nu \pi}{n+2}+o\left(\frac{1}{n}\right)
$$

Proof. See $[6 ; 10$ or 19].

We first reduce the problem to the case of a smooth even function $f(\theta)$.

Lemma 5. Let $f(\theta)$ satisfy Conditions $\mathrm{A}$ and $\mathrm{A}(\alpha)$. To a given $\epsilon<0$ we can find two functions $\hat{f}(\theta)$ and $\bar{f}(\theta)$ satisfying the following conditions.

$$
\begin{aligned}
& \hat{f}(\theta)=m+(1-\cos \theta)^{\alpha}\left[a+b(1-\cos \theta)^{\alpha+1}\right]^{-1}, \\
& \bar{f}(\theta)=m+(1-\cos \theta)^{\alpha}[c+d(1-\cos \theta)],
\end{aligned}
$$

where $a, b, c, d$ are positive constants;

$$
\begin{array}{cc}
\hat{f}(\theta) \leqq f(\theta) \leqq \bar{f}(\theta), & \text { for all } \theta . \\
\left|f^{(2 \alpha)}(0)-\bar{f}^{(2 \alpha)}(0)\right|<\epsilon, \quad\left|f^{(2 \alpha)}(0)-\hat{f}^{(2 \alpha)}(0)\right|<\epsilon . &
\end{array}
$$


Proof. See [6, Chapter 5] or [10] where the proof is given for $\alpha=1$. The proof for general $\alpha$ follows the same lines with only the obvious modifications.

Following Widom, we now reduce the problem to the case of an even trigonometric polynomial.

Lemma 6. Let $f(\theta)$ be an arbitrary even function satisfying Conditions A and $\mathrm{A}(\alpha)$. Moreover, let $f(\theta)$ have $2 \alpha+2$ continuous derivatives in some neighborhood of $\theta=0$. (Note: the functions $\bar{f}(\theta)$ and $\vec{f}(\theta)$ certainly satisfy this additional condition.) To a given small $\epsilon>0$ we can find two even trigonometric polynomials $f_{ \pm \epsilon}(\theta)$ satisfying Conditions $\mathrm{A}$ and $\mathrm{A}(\alpha)$ as well as the following conditions:

$$
\begin{aligned}
f_{-\epsilon}(\theta) & \leqq f(\theta) \leqq f_{+\epsilon}(\theta), \\
\left|f_{ \pm \epsilon}(\theta)-f(\theta)\right| & <\epsilon, \\
f_{ \pm \epsilon}(0) & =m, f_{ \pm \epsilon}^{(2 \alpha)}(0)=\sigma^{2} .
\end{aligned}
$$

for all $\theta$,

Proof. See $[19, \S 2.10]$ where this is proven for $\alpha=1$. The proof for general $\alpha$ follows with only the obvious modifications.

The final elementary reduction is to the special case where

Condition A.1. $f(\theta)$ is an even trigonometric polynomial and all the complex roots of $f(\theta)-m$ are simple.

Lemma 7. Let $f(\theta)$ be an arbitrary even trigonometric polynomial satisfying Conditions $\mathrm{A}$ and $\mathrm{A}(\alpha)$. Then, for sufficiently small $\epsilon>0$ there are two even trigonometric polynomials $g_{ \pm \epsilon}(\theta)$ which satisfy Conditions $\mathrm{A}, \mathrm{A}(\alpha)$, and A.1. Moreover,

$$
\begin{aligned}
g_{ \pm \epsilon}(0) & =m, g_{ \pm \epsilon}^{(2 \alpha)}(0)=\sigma^{2}, \\
g_{-\epsilon}(\theta) & \leqq f(\theta) \leqq g_{+\epsilon}(\theta), \\
\left|g_{ \pm \epsilon}(\theta)-f(\theta)\right| & <\epsilon,
\end{aligned}
$$

for all $\theta$.

Proof. Once more the proof follows the pattern given in [19].

REMARK. Our reason for stating Lemmas 7 and 8 as separate results is simple enough: the proofs in [19] are distinct and it seems logically correct to emphasize this.

Consider such an even trigonometric polynomial.

Let

$$
f(\theta)=\sum_{j=-k}^{k} C_{j} e^{i j \theta}, \quad C_{k} \neq 0,
$$

where, since $f(\theta)$ is even, $C_{-j}=C_{j}, j=1,2, \cdots, k$. Therefore, if $f(\theta)=\lambda$ where $\lambda$ is real, then

$$
f(\bar{\theta})=f(-\theta)=f(-\bar{\theta})=\lambda
$$


also. We now assume $f(\theta)$ to be of the form (2.15) and to satisfy Conditions A, $\mathrm{A}(\alpha)$ and $\mathrm{A} .1$.

Let

$$
P(z ; \lambda)=z^{k}\left\{\sum_{j=-k}^{k} C_{j} z^{j}-\lambda\right\},
$$

i.e., $P(z)$ is the characteristic equation of the eigenvalue problem when that problem is considered as a difference equation (see [13]). If $P(z ; \lambda)=0$ then $P\left(z^{-1} ; \lambda\right)=0$ as well.

The important and basic result of Widom [19] is

Lemma 8. There is an interval $I: m<\lambda<m+\delta$ such that the roots $\theta_{j}$ of $f(\theta)-\lambda$ and the corresponding roots $\rho_{j}=e^{i \theta j}$ of $P(z ; \lambda)$ are simple for $\lambda \in I$. Moreover, $\lambda$ is an eigenvalue of $T_{n}$ if and only if the roots $\rho_{j}$ satisfy

$$
D=\sum_{\tilde{\rho}_{1} \tilde{\rho}_{2} \cdots \tilde{\rho}_{k}} \frac{\left[\tilde{\rho}_{1} \tilde{\rho}_{2} \cdots \tilde{\rho}_{k}\right]^{k+n+1}}{P^{\prime}\left(\tilde{\rho}_{1}\right) \cdots P^{\prime}\left(\tilde{\rho}_{n}\right)} \prod_{s<t}\left(\tilde{\rho}_{s}-\tilde{\rho}_{t}\right)^{2}=0,
$$

where the summation extends over all combinations of $k$ of the zeros $\rho$ of $P(z ; \lambda)$.

Proof. See $[19,(2.17)]$.

The essence of the method used by Widom is that Lemma 8 may be used to reduce the problem for arbitrary $f(\theta)$ of the form (2.15) to a problem like that for the very simplest of such functions, namely

$$
f_{\alpha}(\theta)=(1-\cos \theta)^{\alpha} \text {. }
$$

While Lemma 8 is sufficient for most of our work, we will make use of the following alternative instead. Lemma 9 has the advantage of being naturally related to the theory of difference equations and simplifying some calculations.

LEMmA 9. There is an interval $I: m<\lambda<m+\delta$ such that the roots $\theta_{j}$ of $f(\theta)-\lambda$ and the corresponding roots $\rho_{j}=e^{i \theta j}$ of $P(z ; \lambda)$ are simple for $\lambda \in I$. Moreover, $\lambda$ is an eigenvalue of $T_{n}$ if and only if the roots $\rho_{j}$ satisfy

$$
D_{0}=D_{+} \cdot D_{-}=0 \text {, }
$$

where

$$
\begin{aligned}
& D_{+}=\sum\left[\rho_{1}^{ \pm 1} \rho_{2}^{ \pm 1} \cdots \rho_{k}^{ \pm 1}\right]^{(n+2) / 2} \prod_{s<t}\left(\rho_{s}^{ \pm 1}-\rho_{t}^{ \pm 1}\right), \\
& D_{-}=\sum(-1)^{\nu}\left[\begin{array}{c}
\rho_{1}^{ \pm 1} \rho_{2}^{ \pm 1} \cdots \\
\rho_{k}^{ \pm 1}
\end{array}\right]^{(n+2) / 2} \prod_{s<t}\left(\rho_{s}^{ \pm 1}-\rho_{t}^{ \pm 1}\right),
\end{aligned}
$$

where the summations are taken over all choices of the sign and $\nu=$ the number of minus signs in the term. 
Proof. The interval $I$ is obtained precisely as in [19].

If $\lambda$ is an eigenvalue of $T_{n}$ and $X=\left(X_{j}\right) j=1,2, \cdots, n+1$ associated eigenvector, then the sequence $\left(X_{j}\right)$ satisfies

$$
\sum_{j=-k}^{k} C_{j} X_{r+j}=\lambda X_{r}, \quad r=1,2, \cdots, n+1,
$$

where, of course: $X_{j}=0, j \leqq 0$ and $j \geqq n+1$. In other words, an eigenvalue $\lambda$ and an eigenvector $X$ are obtained if and only if we have a solution of the difference equation (2.18) for which

$$
\begin{array}{lr}
X_{j}=0, & j=0,-1,-2, \cdots,-(k-1), \\
X_{j}=0, & j=n+2, n+3, \cdots, n+k+1 .
\end{array}
$$

Since the $\rho_{i}$ are distinct, every solution of (2.18) is given in the form

$$
X_{r}=\sum_{j=1}^{k}\left(\alpha_{j} \rho_{j}^{r}+\overrightarrow{\beta_{j}} \overrightarrow{\rho_{j}}\right)
$$

(see $[8 ; 13])$. Thus we have

$$
\sum_{j=1}^{k} \alpha_{j} \rho_{j}^{r}+\sum_{j=1}^{k} \beta_{j} \rho_{j}^{-r}=0
$$

for the appropriate values of $r$. Thus the determinant

$$
H=\left|\begin{array}{ll}
A & B \\
C & D
\end{array}\right|=0
$$

where

$$
\begin{aligned}
& A=\left(A_{i j}\right)=\left(\rho_{j}^{-i+1}\right), \\
& B=\left(b_{i j}\right)=\left(\rho_{j}^{i-1}\right), \\
& C=\left(C_{i j}\right)=\left(\rho_{j}^{n+1+i}\right), \\
& D=\left(d_{i j}\right)=\left(\rho_{j}{ }^{-(n+1+i)}\right), \quad i, j=1,2, \cdots, k .
\end{aligned}
$$

If we multiply the $s$ th column $\rho_{\varepsilon}^{-(n+2) / 2}$ and the $(s+k)$ th column by $\rho_{x}^{+(n+2) / 2}$, we obtain

$$
H=H_{0}=\left|\begin{array}{cc}
A_{0} & B_{0} \\
B_{0} & A_{0}
\end{array}\right|=0
$$

where

$$
\begin{aligned}
& A_{0}=\left(A_{i j}\right)=\rho_{j}^{-((n+2) / 2+i-1)}, \\
& B_{0}=\left(B_{i j}\right)=\rho_{j}^{((n+2) / 2-1+i)},
\end{aligned}
$$


However, it is easy to see that

$$
H_{0}=\operatorname{det}\left(A_{0}+B_{0}\right) \cdot \operatorname{det}\left(A_{0}-B_{0}\right) .
$$

Upon expanding these determinants by columns and using the sum formula as well as the well-known formula for a Vandermonde determinant we obtain

$$
\begin{aligned}
& \operatorname{det}\left(A_{0}+B_{0}\right)=D_{+}, \\
& \operatorname{det}\left(A_{0}-B_{0}\right)=D_{-} .
\end{aligned}
$$

It should be noted that

$$
\begin{aligned}
& A_{0}+B_{0}=C_{1}\left|\cos ((n+2) / 2+j-1) \theta_{i}\right|, \quad i, j=1,2, \cdots, k, \\
& A_{0}-B_{0}=C_{2}\left|\sin ((n+2) / 2+j-1) \theta_{i}\right|
\end{aligned}
$$

where $C_{1}$ and $C_{2}$ are constant factors.

Let us agree to order the simple zeros of $f(\theta)-\lambda$ as follows:

$$
\theta_{1}, \theta_{2}, \cdots, \theta_{k}, \theta_{-2}, \cdots, \theta_{-k}
$$

where

$$
\begin{aligned}
\theta_{j} & =-\theta_{-j} \\
\theta_{1} & >0 \\
\operatorname{Im}\left[\theta_{j}\right] & <0, \quad j=2,3, \cdots, k
\end{aligned}
$$

and $\pm \theta_{1}, \pm \theta_{2}, \cdots, \pm \theta_{\alpha}$, are the $2 \alpha$ zeros near $\theta=0$. Then

$$
\left|e^{i \theta j}\right|=\left|\rho_{j}\right|>1, \quad j=2,3, \cdots, k .
$$

The roots $\pm \theta_{\alpha+1}, \cdots, \pm \theta_{k}$ are all bounded away from 0 for $n$ sufficiently large. Hence, $\rho_{\alpha+1}, \rho_{\alpha+2}, \cdots, \rho_{k}$, the roots of $P(z ; \lambda)$ are greater in absolute value than some constant $\mu>1$.

Let us multiply $D_{0}$ by the quantity

$$
\left[\rho_{\alpha+1} \rho_{\alpha+2} \cdots \rho_{k}\right]^{-(n+2)}\left[\prod_{\alpha<s<t}\left(\rho_{s}-\rho_{t}\right)^{-1}\right]^{2} .
$$

We can interpret this as setting

$$
G_{+}=\left[\rho_{\alpha+1} \rho_{\alpha+2} \cdots \rho_{k}\right]^{-(n+2) / 2} \prod_{\alpha<\varepsilon<t}\left(\rho_{s}-\rho_{t}\right)^{-1} \cdot D_{+}
$$

and

$$
G_{-}=\left[\rho_{\alpha+1} \rho_{\alpha+2} \cdots \rho_{k}\right]^{-(n+2) / 2} \prod_{\alpha<\varepsilon<t}\left(\rho_{s}-\rho_{t}\right)^{-1} \cdot D_{-} .
$$

Then, if

$$
G=G_{+} \cdot G_{-}, \quad \text { and } \quad G_{0}=G_{+}^{0} \cdot G_{-}^{0}
$$


where

$$
\begin{aligned}
& G_{+}^{0}=\sum\left(\rho_{1}^{ \pm 1} \rho_{2}^{ \pm 1} \cdots \rho_{\alpha}^{ \pm 1}\right)^{(n+2) / 2} \prod_{<<t \leq \alpha}\left(\rho_{s}^{ \pm 1}-\rho_{t}^{ \pm 1}\right), \\
& G_{-}^{0}=\sum(-1)^{\nu}\left(\rho_{1}^{ \pm 1} \rho_{2}^{ \pm 1} \cdots \rho_{\alpha}^{ \pm 1}\right)^{(n+2) / 2} \prod_{s<t \leq \alpha}\left(\rho_{s}^{ \pm 1}-\rho_{t}^{ \pm 1}\right),
\end{aligned}
$$

we find

Lemma 10. Let $m<\lambda<m+\delta$. Let $\pm \theta_{1}, \pm \theta_{2}, \cdots, \pm \theta_{k}$ be the roots of $f(\theta)-\lambda$. Then $\lambda$ is an eigenvalue of $T_{n}[f]$ if and only if

$$
G=0 \text {. }
$$

Moreover, if $\lambda$ is an eigenvalue of $T_{n}$

$$
G_{0}=O\left(\mu^{(n+2) / 2}\right), \quad G_{+}-G_{+}^{0}=O\left(\mu^{-(n+2) / 2}\right), \quad G_{-}-G_{-}^{0}=O\left(\mu^{-(n+2) / 2}\right) .
$$

Proof. The first statement follows from Lemma 9. The second from the observation that each term of $G_{+}$or $G_{-}$for which the choice of $\rho_{\alpha+1}^{ \pm 1}$ is not $\rho_{\alpha+\rho}^{+1}$ is $O\left(\mu^{-(n+2) / 2}\right)$.

REMARK. We may, if we like, consider $\lambda,-\theta_{1}$, and all the zeros $\pm \theta_{j}$, $j=2,3, \cdots, k$ as functions of $\theta_{1}$. For any annulus

$$
\frac{B_{0}}{n}<\left|\theta_{1}\right|<\frac{B_{1}}{n} \text {. }
$$

in which the roots $\pm \theta_{j}$ are distinct, all these functions are analytic functions of $\theta_{1}$. Hence $G, G_{+}^{0}, G_{-}^{0}$, and $G_{0}$ are all analytic functions of $\theta_{1}$.

The line of attack is now clear. We must now study the zeros of $G_{0}$ (the zeros of $G_{+}^{0}$ and $G_{-}^{0}$ ) and obtain an estimate from below for $\left|G_{0}\right|$ away from these zeros. Then Rouché's theorem and Theorem 1 will enable us to claim that the zeros of $G$ are essentially the zeros of $G_{0}$. In the next section we will carry out this program for the case $\alpha=2$.

The form of $G_{0}$ justifies our earlier remark on the relationship of the general problem to that for the function

$$
f_{\alpha}(\theta)=(1-\cos \theta)^{\alpha} \text {. }
$$

Let us pursue this line of thought a bit further. We may write $f(\theta)$ in the form

$$
f(\theta)=m+\beta(1-\cos \theta)^{\alpha}+\sum_{\alpha+1}^{k} \beta_{j}(1-\cos \theta)^{j} .
$$

Then, for $n$ sufficiently large, Theorem 1 shows that if $\lambda$ is an eigenvalue of $T_{n}$

$$
f_{*}(\theta)=m+\beta f_{\alpha}(\theta)=\lambda+O\left(n^{-(2 \alpha+2)}\right) .
$$


Hence, for $n$ large enough, the zeros of $G_{0}$ are like the zeros of $G$ for the function $f_{*}(\theta)$. However, as we shall see, for $f_{*}(\theta)$ we have a much stronger form of Lemma 3. Then, given the appropriate estimate on $G_{0}$ we should be able to claim this stronger form of Lemma 3 for all functions $f(\theta)$ satisfying Conditions $\mathrm{A}$ and $\mathrm{A}(\alpha)$. We conjecture the truth of this stronger estimate. We now prove the above remark about the function $f_{*}(\theta)$. We require the well-known

LEMMA 11. Let $C_{n}$ be the matrix of order $n$ given by

$$
C_{i j}=\left(\delta_{i-1, j}+\delta_{i+1, j}\right) \text {, }
$$

i.e.,

$$
C_{n}=\left[\begin{array}{lllllll}
0 & 1 & & & & & \\
1 & 0 & 1 & & & & \\
& & & & 1 & 0 & 1 \\
& & & & & 1 & 0
\end{array}\right] .
$$

Then, the eigenvalues of $C_{n}$ are $\mu_{k}=2 \cos \pi k /(n+1), k=1,2, \cdots, n$.

Proof. See [16].

Lemma 12. Let $f(\theta)$ be an even polynomial of degree $k$. Let $\lambda$ be the minimal eigenvalue of $T_{n}$. Then

$$
\lambda \geqq f\left(\frac{\pi}{n+2 k}\right) .
$$

Proof. We write $f(\theta)$ in its real form, i.e.

$$
f(\theta)=\sum_{j=0}^{k} d_{j} \cos j \theta .
$$

Consider the matrix $F$ of order $n+2 k-1$ given by

$$
F=\sum_{j=0}^{k} d_{j}\left[(1 / 2) A_{n+2 k-1}\right]^{j} .
$$

One sees by direct computation that one obtains $T_{n}[f]$ from $F$ by deleting the first and last $k-1$ rows and columns. Thus by Lemmas 1 and 11

$$
\lambda \geqq \operatorname{Min}_{\|x\|=1}\left(\frac{X, F X}{X, X}\right)=f\left(\frac{\pi}{n+2 k}\right) .
$$

Thus, if indeed it is sufficient to consider $G_{0}$ instead of $G$, we may consider the roots of the function $G_{0}$ obtained from $f_{*}(\theta)$. Hence we are led to the

Conjecture. Let $f(\theta)$ satisfy Conditions $\mathrm{A}$ and $\mathrm{A}(\alpha)$. Let $\lambda$ be the minimal eigenvalue of $T_{n}[f]$. 


$$
\lambda \geqq f\left[\frac{\pi}{n+2 \alpha-1}+o\left(\frac{1}{n}\right)\right] .
$$

3. The case $\alpha=2$. Let $f(\theta)$ be an even trigonometric polynomial of the form (2.15) satisfying Conditions $\mathrm{A}, \mathrm{A}(\alpha)$ with $\alpha=2$, and Condition A.1. Then $f(\theta)-\lambda$ has four roots near $\theta=0$; two are real, say $\pm \theta$, and two are pure imaginary numbers, say $\pm i \beta$.

We compute $G_{0}, G_{+}^{0}$ and $G_{-}^{0}$ and discuss the roots of $G_{0}$. By direct calculations we obtain,

$$
\begin{aligned}
G_{+}^{0}=C_{1}\left\{\cos \frac{n+3}{2} \theta \cos \frac{\theta}{2} \sinh \frac{n+3}{2} \beta \sinh \frac{\beta}{2}\right. \\
\left.+\cosh \frac{n+3}{2} \beta \cosh \frac{\beta}{2} \sin \frac{n+3}{2} \theta \sin \frac{\theta}{2}\right\}, \\
G_{-}^{0}=C_{2}\left\{\sin \frac{n+3}{2} \theta \cos \frac{\theta}{2} \cosh \frac{n+3}{2} \beta \sinh \frac{\beta}{2}\right.
\end{aligned}
$$

$$
\left.-\sinh \frac{n+3}{2} \beta \cosh \frac{\beta}{2} \sin \frac{\theta}{2} \cos \frac{n+3}{2} \theta\right\},
$$

$$
\begin{aligned}
G_{0}=2\{\sin \theta \sinh \beta & {[\cos (n+3) \theta \cosh (n+3) \beta-1] } \\
& -\sin (n+3) \theta \sinh (n+3) \beta[\cos \theta \cosh \beta-1]\} .
\end{aligned}
$$

Consider the functions

$$
\begin{aligned}
& H_{+}(t)=\operatorname{ctn} t \theta \cdot \tanh t \beta+\tan \frac{1}{2} \theta \cdot \operatorname{ctnh} \frac{1}{2} \beta, \\
& H_{-}(t)=\tan t \theta \cdot \operatorname{ctnh} t \beta-\tan \frac{1}{2} \theta \operatorname{ctn} \frac{1}{2} \beta .
\end{aligned}
$$

$G_{+}^{0}$ and $G_{-}^{0}$ have zeros if and only if $H_{+}(t)$ and $H_{-}(t)$ respectively have zeros for $t=(n+3) / 2$. The function $H_{+}(t) \geqq 0$ for $0 \leqq t \theta \leqq \pi / 2$. Hence $G_{+}^{0}$ has no zeros for $0<\theta \leqq \pi /(n+3)$. Since

$$
\dot{H}_{-}(t)=\frac{1}{2} \sec ^{2} t \theta \cdot \operatorname{csch}^{2} t \beta[\theta \sinh 2 t \beta-\beta \sin 2 t \theta]
$$

and $\sin \theta \leqq \theta, \beta \leqq \sinh \beta$ for $\theta, \beta>0$ we find

$$
\dot{H}_{-}(t)=\frac{1}{4 t} \sec ^{2} t \theta \cdot \operatorname{csch}^{2} t \beta\{2 t \theta \sinh 2 t \beta-2 t \beta \sin 2 t \theta\}>0 .
$$

Comparing $H_{+}$and $H_{-}$, we find that this argument also gives

$$
H_{+}(t) \leqq 0 \text {. }
$$


From this we conclude:

There are no roots of $G_{+}^{0}$ or $G_{-}^{0}$, and hence of $G_{0}$, in the interval

$$
0<\theta<\frac{\pi}{n+3} .
$$

Moreover, since $\sin \theta / 2 \cdot \sinh \beta / 2>0$ and $\tanh t \beta$ and $\operatorname{ctnh} t \beta$ are bounded, positive quantities, $G_{+}^{0}$ has one and only one zero in every interval

$$
\frac{(2 j-1) \pi}{n+3}<\theta<\frac{2 j \pi}{n+3}, \quad j=1,2, \cdots
$$

while $G_{-}^{0}$ has one and only one zero in every interval

$$
\frac{2 j \pi}{n+3}<\theta<\frac{2 j+1}{(n+3)} \pi, \quad j=1,2, \cdots .
$$

In order to study the zeros of $G_{0}$ in greater detail we turn to an analysis of the function

$$
H(t)=\frac{\sin t \theta \cdot \sinh t \beta}{1-\cos t \theta \cdot \cosh t \beta}, \quad \quad t>\frac{\pi}{\theta}
$$

and its crossings with $H(1)$. As in the previous discussion, $G_{0}$ has a root if and only if $H(n+3)=H(1)$. Since

$$
\dot{H}(t)=\frac{(\beta \sin t \theta-\theta \sinh t \beta)(\cosh t \beta-\cos t \theta)}{(1-\cos t \theta \cdot \cosh t \beta)^{2}},
$$

we conclude (exactly as with $\left.H_{-}(t)\right)$ that

$$
\dot{H}(t) \leqq 0 .
$$

Since $H(j \pi / \theta)=0$ and $H(1)>0$ we may conclude that the roots of $G_{0}$ which correspond to roots of $G_{+}^{0}$ fall in the intervals

$$
\frac{4 j-1}{2(n+3)} \pi<\theta<\frac{2 j \pi}{n+3} .
$$

Moreover, since $H(1)=O\left(n^{2}\right)$ and not $o\left(n^{2}\right)$ and for fixed $j, j=1,2, \cdots$

$$
H\left(\frac{4 j+1}{2 \theta} \pi\right)=\sinh \frac{4 j+1}{2} \frac{\beta}{\theta}=O(1),
$$

the roots of $G_{0}$ corresponding to roots of $G_{-}^{0}$ fall in the intervals

$$
\frac{2 j \pi}{n+3}<\theta<\frac{4 j+1}{2} \pi \text {. }
$$


As was noted in $\S 1$, the roots $\theta$ and $\beta$ are asymptotic to the roots of $(1-\cos x)^{2}=\lambda$, i.e.

$$
1-\cos \theta=\lambda^{1 / 2}
$$

and

$$
1-\cosh \beta=-\lambda^{1 / 2} \text {. }
$$

An investigation of this case shows that we have $\beta \sim \theta$, and we shall now use this relationship.

Having determined that $\theta=x /(n+3)$ where $x=O(1)$ we write

$$
\beta=\theta=\frac{x}{n+3}
$$

and substitute into the expressions (3.1a) and (3.1b). A direct computation yields the following results.

The roots of $G_{+}^{0}$ are asymptotically (to order $1 / n$ ) the roots of

$$
\tan \frac{(n+3) \theta}{2}=-\tanh \frac{(n+3) \theta}{2} .
$$

The roots of $G_{-}^{0}$ are asymptotically (to order $1 / n$ ) the roots of

$$
\tan \frac{(n+3) \theta}{2}=\tanh \frac{(n+3) \theta}{2} \text {. }
$$

Following the course laid out in the previous section we obtain

TheOREM 3. Let $f(\theta)$ be a function which satisfies Conditions A and $\mathrm{A}(\alpha)$ with $\alpha=2$. Let $\lambda_{v, n}$ be the eigenvalues of $T_{n}[f]$ ordered in nondecreasing order. Then for fixed $\nu, \nu=1,2, \cdots$, we have, as $n \rightarrow \infty$,

$$
\lambda_{\nu, n}=f\left[\frac{(2 \nu+1) \pi+E_{\nu}}{2(n+3)}+o\left(\frac{1}{n}\right)\right],
$$

or, equivalently

$$
\lambda_{\nu, n}=m+\frac{1}{4 !}\left\{\frac{(2 \nu+1) \pi+E_{\nu}}{2(n+3)}\right\}^{4} f^{4}(0)+o\left(n^{-4}\right) .
$$

Moreover, the $E$, are determined from the equation

$$
\tan \left(\frac{(2 \nu+1) \pi+E_{\nu}}{4}\right)=(-1)^{\nu} \tanh \left(\frac{(2 \nu+1) \pi+E_{\nu}}{4}\right) .
$$

In fact, 


$$
\begin{array}{ll}
\frac{3 \pi+E_{1}}{4}=2.3650, & \frac{7 \pi+E_{3}}{4}=5.4978, \\
\frac{5 \pi+E_{2}}{4}=3.9266, & \frac{9 \pi+E_{4}}{4}=7.0686 .
\end{array}
$$

Proof. Using Lemmas 5, 6 and 7 we see we need only consider even trigonometric polynomials which also satisfy Condition A.1. The final result then follows from Lemma 10, the above estimates on the roots of $G_{0}$ and the form (3.1c) of $G_{0}$ which enables us to apply Rouche's theorem. The exact numerical values are obtained from Jahnke and Emde $[9$, p. 31] where a thorough discussion of the roots of equation 3.9 is to be found.

4. The Conditions $\mathrm{B}$ and $\mathrm{B}(\alpha)$. Consider a function $f(\theta)$ which satisfies Conditions $B$ and $B(\alpha)$. The argument of Lemma 4 may be repeated verbatim at either of the points $\theta_{0}$ or $-\theta_{0}$. Hence we obtain

$$
\lambda_{1, n} \leqq m+O\left(\frac{1}{n}\right)^{2 \alpha} \text {. }
$$

To obtain a lower estimate we proceed as in Lemma 3 . Let $X=\left(X_{j}\right) j=0,1$, $\cdots, n$ be the normalized eigenvector associated with the minimal eigenvalue $\lambda_{1, n}$. Since $f(\theta)$ is even, $T_{n}[f]$ is a real symmetric matrix and the $X_{j}$ are real. Hence, if $g(\theta)=\sum_{j=0}^{n} X_{j} e^{i j \theta}$, the function $|g(\theta)|^{2}$ is even. Thus

$$
\lambda_{1, n}=\frac{1}{2 \pi} \int_{-\pi}^{\pi} f(\theta)|g|^{2} d \theta=\frac{1}{\pi} \int_{0}^{\pi} f(\theta)|g|^{2} d \theta .
$$

Let

$$
f_{0}(\phi)=f\left(\frac{\phi}{2}+\theta_{0}\right), \quad h(\phi)=g\left(\frac{\phi}{2}+\theta_{0}\right)
$$

then

$$
\lambda_{1, n}=\frac{1}{2 \pi} \int_{-\pi}^{\pi} f_{0}(\phi)|h|^{2} d \phi .
$$

We notice that $f_{0}(\phi)$ is a function satisfying Conditions $\mathrm{A}$ and $\mathrm{A}(\alpha)$ while $|h|^{2}$ satisfies

$$
\operatorname{Max}|h|^{2}=\operatorname{Max}|g|^{2} \leqq n+1 .
$$

We may now repeat the argument of Lemma 3 to obtain

$$
\lambda_{1, n} \geqq m+O\left[\left(\frac{1}{n}\right)^{2 \alpha}\right] \text {. }
$$

Hence we have proved the analogous theorem to Theorem 1. Namely 
TheOREM 4. Let $f(\theta)$ satisfy Conditions $\mathrm{B}$ and $\mathrm{B}(\alpha)$. Let $\lambda_{1, n}$ be the minimal eigenvalue of $T_{n}[f]$. Then

$$
\lambda_{1, n}=m+O\left(\frac{1}{n}\right)^{2 \alpha}
$$

where $O$ cannot be replaced by 0 .

Proceeding exactly as in $\$ 1$ we could now prove the appropriate analogs of Lemmas 5, 6 and 7. Indeed, if the expression $(1-\cos \theta)^{\alpha}$ is replaced (both in the statements of the results and in the proofs as in Widom [19]) by $\left[1-\cos \left(\theta-\theta_{0}\right)\right]^{\alpha}\left[1-\cos \left(\theta+\theta_{0}\right)\right]^{\alpha}$, the exact analogs hold. We may sum up these approximation argument as follows:

It is sufficient to consider the case where $f(\theta)$ is a trigonometric polynomial which satisfies Conditions $\mathrm{B}, \mathrm{B}(\alpha)$ and the complex roots of $f(\theta)-m$ are simple.

Furthermore the argument. of Lemma 9 depends only on the fact that all roots of $f(\theta)-\lambda$ are distinct. Hence an analog of Lemma 9 applies to this case as well. In fact, only the first sentence needs to be changed to allow for two intervals about $\pm \theta_{0}$.

Consider now the simple roots of $f(\theta)-\lambda$. Exactly four of them will be real and these will occur in pairs; say $\pm \theta_{1}, \pm \theta_{\alpha+1}$. Thus we may order these simple zeros of $f(\theta)-\lambda$ as follows:

$$
\theta_{1}, \theta_{2}, \cdots, \theta_{k}, \theta_{-1}, \theta_{-2}, \cdots, \theta_{-k}
$$

where

$$
\begin{aligned}
& \qquad \begin{aligned}
& \theta_{j}=-\theta_{-j}, \\
& \theta_{1}=\theta_{0}+\psi_{1}>0, \quad \theta_{\alpha+1}=\theta_{0}-\psi_{2}>0, \\
& I_{m}\left(\theta_{j}\right)<0, \\
& \text { the roots } \theta_{2} \theta_{3} \cdots \theta_{\alpha} \text { and } \theta_{\alpha+2} \theta_{\alpha+3} \cdots \theta_{2 \alpha}
\end{aligned}
\end{aligned}
$$

are the roots "near" $\theta_{0}$. If we now repeat the essential argument of Lemma 10 we obtain

LEMMA 13. Let $\rho_{j}=e^{i \theta_{j}}$ and let

$$
\begin{aligned}
& \Gamma_{+}=\left[\rho_{2 \alpha+1} \rho_{2 \alpha+2} \cdots \rho_{k}\right]^{-(n+2) / 2} \prod_{2 \alpha<\varepsilon<t}\left(\rho_{s}-\rho_{t}\right)^{-1} \cdot D_{+}, \\
& \Gamma_{-}=\left[\rho_{2 \alpha+1} \rho_{2 \alpha+2} \cdots \rho_{k}\right]^{-(n+2) / 2} \prod_{2 \alpha<<<t}\left(\rho_{z}-\rho_{t}\right)^{-1} \cdot D_{-} \text {, } \\
& \Gamma_{+}^{0}=\sum\left[\rho_{1}^{ \pm 1} \rho_{2}^{ \pm 1} \cdots \rho_{2 \alpha}^{ \pm 1}\right]^{(n+2) / 2} \prod_{\varepsilon<t \leq 2 \alpha}\left(\rho_{s}^{ \pm 1}-\rho_{t}^{ \pm 1}\right), \\
& \Gamma_{-}^{0}=\sum(-1)^{\nu}\left[\rho_{1}^{ \pm 1} \rho_{2}^{ \pm 1} \cdots \rho_{2 \alpha}^{ \pm 1}\right]^{(n+2) / n} \prod_{<<t \leq 2 \alpha}\left(\rho_{t}^{ \pm 1}-\rho_{t}^{ \pm 1}\right) \text {. }
\end{aligned}
$$

Let 


$$
\Gamma=\Gamma_{+} \cdot \Gamma_{-} \text {and } \Gamma_{0}=\Gamma^{0} \cdot \Gamma_{-}^{0} \text {. }
$$

Let $m<\lambda<m+\delta$. Then $\lambda$ is an eigenvalue of $T_{n}[f]$ if and only if

$$
\Gamma=0 \text {. }
$$

Moreover, if $\lambda$ is an eigenvalue of $T_{n}[f]$

$$
\Gamma_{0}=O\left(\mu^{-(n+2) / 2}\right), \quad \Gamma_{ \pm}-\Gamma_{ \pm}^{0}=O\left(\mu^{-(n+2) / 2}\right),
$$

where $\mu$ satisfies

$$
1<\mu \leqq\left|\rho_{j}\right|, \quad j>2 \alpha .
$$

Hence we may once more concentrate on the zeros of $\Gamma_{0}$ and apply Rouché's theorem after obtaining the appropriate estimate.

Let us now consider the case $\alpha=1$. By an argument just like the one of $\$ 1$ we find that $\theta_{1}$ and $\theta_{\alpha+1}$ are asymptotic to the positive roots of $\left[1-\cos \left(\theta-\theta_{0}\right)\right]\left[1-\cos \left(\theta+\theta_{0}\right)\right]=\lambda$. Note that Theorem 4 implies that $\psi_{1}$ and $\psi_{2}$ are $O(1 / n)$ and not $o(1 / n)$. Using this fact we obtain $\psi_{1}=\psi_{2}+O(1 / n)^{2}$. By a direct, though tedious, calculation (in which we use the asymptotic relationship $\psi_{1}=\psi_{2}=\psi$ ) we find

$$
\begin{aligned}
& \Gamma_{+}^{0}=C\{\sin (n+3) \psi+\sin \psi\} \sin \theta_{0}, \\
& \Gamma_{-}^{0}=C\{\sin (n+3) \psi-\sin \psi\} \sin \theta_{0} .
\end{aligned}
$$

Thus, to order $1 / n, \Gamma_{+}^{0}$ and $\Gamma_{-}^{0}$ have the same roots; namely $\pi \nu /(n+3)$. Thus we have the first order characterization of the minimal eigenvalues of $f(\theta)$.

TheOREM 5. Let $\lambda_{\nu, n}$ be the eigenvalues of $T_{n}[f]$ ordered in increasing order, i.e. we count multiple roots only once. Then for fixed $\nu, \nu=1,2, \cdots$ we have, as $n \rightarrow \infty$

$$
\lambda_{\nu, n}=f\left[\theta_{0}+\frac{\pi \nu}{n}+o\left(\frac{1}{n}\right)\right] .
$$

Moreover, for each $\nu$ there are two linearly independent eigenvectors corresponding to this asymptotic value.

Proof. It is only necessary to verify that we may apply Rouchés theorem in this case. This is apparent from the form of $\Gamma_{+}^{0}$ and $\Gamma_{-}^{0}$. Indeed all of the details have been carried out by Widom in [19].

5. Block Toeplitz matrices. A block Toeplitz matrix $M$ is simply a matrix of the form $\left(C_{8-r}\right)$ where the $C_{j}$ are themselves square matrices and $C_{j}^{*}=C_{-j}$ where $C_{j}^{*}$ is the adjoint of $C_{j}$. The corresponding function $f(\theta)$ is then a matrixvalued function which is Hermitian. In the notation of the "direct product" (see $[12, \S 7]$ ) we write 


$$
M=A_{0} \otimes I_{n+1}+\sum_{j=1}^{n}\left\{C_{j} \otimes J_{n+1}^{j}+C_{j}^{*} \otimes\left(J_{n+1}^{*}\right)^{j}\right\}
$$

where $J_{n+1}$ is the square matrix of order $n+1$ given by $J_{n+1}=\left(\delta_{i, j-1}\right)$, i.e.

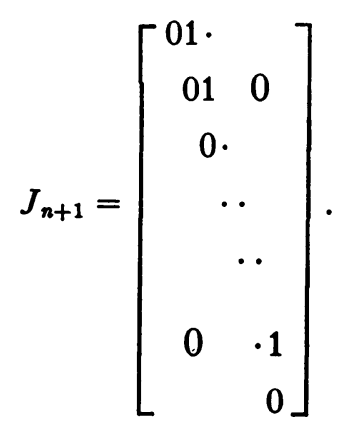

The function $f(\theta)$ is given by

$$
f(\theta) \sim A_{0}+\sum_{j=1}^{\infty}\left\{C_{j} e^{i j \theta}+C_{j}^{*} e^{-i j \theta}\right\} .
$$

Let $f(\theta)$ be of order $N$. Let $X$ be any $(n+1) N$ dimensional vector written in "block" form, i.e.

$$
X=\left(X_{k}\right),
$$$$
k=0,1, \cdots, n
$$

where each $X_{k}$ is itself an $N$-vector. As in Lemma 2, if

$$
G(\theta)=\sum_{j=0}^{n} X_{j} e^{-i j \theta}
$$

we have

$$
\left(X, T_{n}[f] X\right)=\frac{1}{2 \pi} \int_{-\pi}^{\pi} G^{*}(\theta) f(\theta) G(\theta) d \theta .
$$

We now turn to the study of the minimal (or maximal) eigenvalues of $T_{n}[f]$ where $f(\theta)$ is an Hermitian-matrix valued function which is continuous and of period $2 \pi$.

Since $f(\theta)$ is an Hermitian matrix for every value of $\theta$ there is a function $\mu(\theta)$ defined by

$$
\mu(\theta)=\text { minimum eigenvalue of } f(\theta) .
$$

Moreover, there is an eigenvector of norm 1, say $v(\theta)=\left(v_{j}(\theta)\right)$, so that

$$
f(\theta) v(\theta)=\mu(\theta) v(\theta)
$$

and

$$
v^{*} f v=\mu(\theta) v^{*} v=\mu(\theta) .
$$


If $\mu(\theta)$ and $v(\theta)$ are continuous we may immediately conclude

$$
\lambda \geqq \underset{\theta}{\operatorname{Min}} \mu(\theta)
$$

where $\lambda$ is any eigenvalue of $T_{n}[f]$. Moreover, $\min \lambda \Rightarrow \operatorname{Min}_{\theta} \mu(\theta)$ as $n \rightarrow \infty$.

We turn now to the case where $\mu(\theta)$ and $v(\theta)$ are "nicely" behaved and $\mu(\theta)$ satisfies Conditions $\mathrm{A}$ and $\mathrm{A}(\alpha)$ or $\mu(\theta)$ satisfies Conditions $\mathrm{B}$ and $\mathrm{B}(\alpha)$.

We require certain estimates on behaviour of the functions $|\phi(\theta)|^{2}$ which provide the minimum in Lemma 2 in the scalar case.

LEMMA 14. Let $f(\theta)$ be a real valued continuous function which satisfies Conditions A and $\mathrm{A}(\alpha)$. Let $X=\left(X_{j}\right), j=0,1, \cdots, n$ be the eigenvector of $T_{n}[f]$ associated with the minimal eigenvalue $\lambda_{1, n}$. Let $\eta>0$ be a fixed number such that

$$
\begin{array}{lr}
f(\theta) \geqq f(\eta) & \text { for } \eta \leqq \theta \leqq \pi, \\
f(\theta) \geqq f(-\eta) & \text { for }-\pi \leqq \theta \leqq-\eta .
\end{array}
$$

Moreover we require that $\eta$ be so small that there exists a constant $A>0$ such that

$$
A \theta^{2 \alpha} \leqq f(\theta)-m,
$$$$
|\theta| \leqq \eta \text {. }
$$

Let $\phi(\theta)=\sum_{j=0}^{n} X_{j} e^{i j \theta}$ and $\sum\left|X_{j}\right|^{2}=1$. Then

$$
\int_{\eta \leq|\theta| \leq \pi}|\phi|^{2} d \theta \leqq O\left(\frac{1}{n}\right)^{2 \alpha}
$$

and

$$
\int_{\eta \leq|\theta| \leq \pi}|\phi|^{2} d \theta=o\left(\frac{1}{n}\right)^{2 \alpha} \quad \text { for } \alpha=1,2
$$

Finally

$$
\int_{-,}^{n} \theta^{\gamma}|\phi|^{2} d \theta=O\left(\frac{1}{n}\right)^{\gamma}, \quad 0 \leqq \gamma \leqq 2 \alpha .
$$

Proof. By Theorem 1

$$
\int_{-\pi}^{\pi}[f(\theta)-m]|\phi|^{2} d \theta=O\left(\frac{1}{n}\right)^{2 \alpha} .
$$

Therefore

$$
\int_{\eta \leq|\theta| \leq \pi}[f(\theta)-m]|\phi|^{2} d \theta=O\left(\frac{1}{n}\right)^{2 \alpha}
$$

or

$$
[f(\eta)-m] \int_{\eta \leq|0| \leq x}|\phi|^{2} d \theta=O\left(\frac{1}{n}\right)^{2 \alpha}
$$


Since $[f(\eta)-m]^{-1}=O(1)$ we have verified (5.11a).

For $\alpha=1$ or 2 we know from Theorems 2 and 3 that the expression

$$
\int_{\text {is }|\theta| \leq \pi}[f(\theta)-m] \cdot|\phi|^{2} d \theta
$$

is independent of $f(\theta)$ to order $(1 / n)^{2 \alpha}$. Hence (5.11b) follows at once.

In the same way we find

$$
0 \leqq \int_{1 / n \leqq|\theta| \leq n}[f(\theta)-m] \cdot|\phi|^{2} d \theta=I_{1}=O\left(\frac{1}{n}\right)^{2 \alpha} .
$$

However, by the choice of $\eta$ we have

$$
I_{1} \geqq A \int_{1 / n \leqq|\theta| \leq \eta} \theta^{2 \alpha}|\phi|^{2} d \theta \geqq A\left(\frac{1}{n}\right)^{2 \alpha-\gamma} \int_{1 / n \leq|\theta| \leq \eta} \theta^{\gamma}|\phi|^{2} d \theta .
$$

Hence we may conclude

$$
\int_{1 / n \leq|\theta| \leq \eta} \theta^{\gamma}|\phi|^{2} d \theta=O\left(\frac{1}{n}\right)^{\gamma} .
$$

Finally we use the fact that $|\phi|^{2} \leqq n+1$ and obtain

$$
\int_{|\theta| \leq 1 / n} \theta^{\gamma}|\phi|^{2} d \theta \leqq \frac{(n+1)}{\gamma+1}\left(\frac{1}{n}\right)^{\gamma+1}=O\left(\frac{1}{n}\right)^{\gamma} .
$$

Thus (5.11b) is proved by adding (5.12a) and (5.12b).

In precisely the same manner we obtain an analogous result for functions satisfying Conditions $\mathrm{B}$ and $\mathrm{B}(\alpha)$.

THEOREM 6. Let $f(\theta)$ be a matrix-valued function which is Hermitian and periodic with period $2 \pi$. Let $\mu(\theta)$ be the minimal eigenvalue for every value of $\theta$ and $v(\theta)$ be an associated normalized eigenvector so that (5.7a) and (5.7b) hold. Let $\mu(\theta)$ satisfy Conditions $\mathrm{A}$ and $\mathrm{A}(\alpha)$. Let $v(\theta)$ have $2 \alpha$ continuous derivatives in a neighborhood of $\theta=0$. Let $\lambda_{1, n}$ be the minimal eigenvalue of $T_{n}[f(\theta)]$. Then

$$
\lambda_{1, n}=\mu(0)+O\left(\frac{1}{n}\right)^{2 \alpha} .
$$

Furthermore, if $\alpha=1$ we have

(5.13b) $\lambda_{1, n}=\mu\left(\frac{\pi}{n}+o\left(\frac{1}{n}\right)\right)=\mu(0)+\frac{1}{2}\left(\frac{\pi}{n}\right)^{2} \mu^{(2)}(0)+o\left(\frac{1}{n}\right)^{2}$.

Finally, if $\alpha=2$ we have

$$
\lambda_{1, n}=\mu\left(\frac{4.73}{n}+o\left(\frac{1}{n}\right)\right)=\mu(0)+\frac{1}{4 !}\left(\frac{4.73}{n}\right)^{4} \mu^{(4)}(0) .
$$


Proof. Let $H(\theta)$ be the vector-valued trigonometric polynomial of the form

$$
H(\theta)=\sum_{j=0}^{2 \alpha} H_{j} e^{-i j \theta}
$$

which satisfies

$$
\left.\left(\frac{d}{d \theta}\right)^{k} H(\theta)\right|_{\theta=0}=\left.\left(\frac{d}{d \theta}\right)^{k} v(\theta)\right|_{0=0}, \quad k=0,1, \cdots, 2 \alpha .
$$

One can easily verify that these $N$ sets of linear equations always have a unique solution. Thus we have

$$
\begin{aligned}
H^{*}(0) \cdot H(0) & =1, \\
H^{*}(\theta) f(\theta) H(\theta) & =\mu(\theta)+r(\theta),
\end{aligned}
$$

where

$$
r(\theta)=o\left(\theta^{2 \alpha}\right),
$$$$
\text { for }|\theta|<\delta
$$

for some $\delta>0$.

Let $g(\theta)$ be the scalar function of the form

$$
g(\theta)=\sum_{j=0}^{n-2 \alpha} \gamma_{j} e^{-i j \theta}
$$

which satisfies

$$
\frac{1}{2 \pi} \int_{-\pi}^{\pi}|g|^{2} d \theta=1
$$

and

$$
\lambda_{1, n-2 \alpha}(\mu)=\min _{0} \frac{1}{2 \pi} \int_{-\pi}^{\pi} \mu(\theta)|g|^{2} d \theta .
$$

Let $G(\theta)=H(\theta) g(\theta)$. Let

$$
\begin{aligned}
& I_{1}=\frac{1}{2 \pi} \int_{-\pi}^{\pi} G^{*} f(\theta) G d \theta \\
& I_{2}=\frac{1}{2 \pi} \int_{-\pi}^{\pi} G^{*} G d \theta=\frac{1}{2 \pi} \int_{-\pi}^{\pi} H^{*} H \cdot|g|^{2} d \theta .
\end{aligned}
$$

By (5.3b) and Lemma 1 we have

$$
\lambda_{1, n} \leqq \frac{I_{1}}{I_{2}} .
$$


In virtue of the estimates of the preceding lemma and Theorems 1, 2 and 3 we immediately obtain

$$
\lambda_{1, n} \leqq \frac{\text { Right-hand side of (5.13) }}{I_{2}} .
$$

However, using (5.15a) and the preceding lemma we readily verify that

$$
I_{2} \geqq 1-O\left(\frac{1}{n}\right)
$$

Hence we have one-half of our result.

The reverse inequality is trivial since

$$
G^{*} f(\theta) G \geqq \mu(\theta) G^{*} G
$$

for all $\theta$. Hence

$$
\lambda_{1, n} \geqq \frac{\frac{1}{2 \pi} \int_{-\pi}^{\pi} \mu(\theta) G^{*} G d \theta}{\frac{1}{2 \pi} \int_{-\pi}^{\pi} G^{*} G d \theta}
$$

Since $G^{*} G$ is a positive scalar polynomial (indeed a sum of terms of the form $|g|^{2}$, where $|g|^{2}$ are scalar polynomials) the conclusion follows from Theorems 1,2 and 3 .

In exactly the same way one can prove the following result for the case when $\mu(\theta)$ satisfies Conditions $B$ and $B(\alpha)$.

THEOREM 7. Let $f(\theta)$ be a matrix-valued function which is Hermitian and periodic with period $2 \pi$. Let $\mu(\theta)$ and $v(\theta)$ be the minimal eigenvalue and eigenvector functions so that (5.7a) and (5.7b) hold. Let $\mu(\theta)$ satisfy Conditions B and $\mathrm{B}(\alpha)$. Let $v(\theta)$ have $2 \alpha$ continuous derivatives in a neighborhood of $\theta=\theta_{0}$. Let $\lambda_{1, n}$ be the minimal eigenvalue of $T_{n}[f(\theta)]$. Then

$$
\lambda_{1, n}=\mu\left(\theta_{0}\right)+O\left(\frac{1}{n}\right)^{2 \alpha} .
$$

Furthermore, if $\alpha=1$ we have

$$
\lambda_{1, n}=\mu\left(\theta_{0}+\frac{\pi}{n}+o\left(\frac{1}{n}\right)\right) .
$$

We close this section with a theorem on the continuity of $\lambda_{1, n}$.

THEOREM 8. Let $f(\theta ; t)$ be a matrix-valued function of $\theta$ which is Hermitian and periodic with period $2 \pi$. Let $f(\theta ; t)$ be "smooth" in $t$, say, continuously differentiable for $t_{0} \leqq t \leqq t_{1}$. Suppose moreover that $\mu(\theta ; t)$, the minimal eigenvalue 
for every $\theta$, and $v(\theta)$, the corresponding normalized eigenvector are also "smooth" in $t$. Finally suppose $\mu(\theta ; t)$ satisfies Condition A for $t_{0} \leqq t \leqq t_{1}$ and

(a) satisfies Condition A(1) for $t_{0}<t \leqq t_{1}$,

(b) satisfies Condition A(2) for $t=t_{0}$.

Then

$$
\mu\left(\frac{\pi}{n}+o\left(\frac{1}{n}\right)\right) \leqq \lambda_{1, n} \leqq \mu\left(\frac{4.73}{n}+o\left(\frac{1}{n}\right)\right)
$$

for all $t$ in $\left[t_{0}, t_{1}\right]$.

Proof. Clearly $\lambda_{1, n}$ is continuous in $t$. Since

$$
\lambda_{1, n}=\mu(\theta(t), t)
$$

$\theta(t)$ must also be a continuous function of $t$. The theorem follows at once.

6. A special case. In our earlier work we sought to estimate the spectral norm of the following special block Toeplitz matrices.

Let $A_{n}$ be the $2 n \times 2 n$ matrix of the form

$$
A_{n}=\left[\begin{array}{lllllll}
0 & R & & & & \\
R^{*} & 0 & R & & & \\
& & & \cdot & & \\
& & & \cdot & \\
& & & & \cdot & R \\
& & & & R^{*} & 0
\end{array}\right],
$$

where $R$ is a real $2 \times 2$ matrix. If $R^{*} R=R R^{*}$ the problem is completely solved by the methods of matrix direct-products. In that case all the eigenvalues of $A_{n}$ are given by

$$
\mu_{o}^{(k)}=2\left|\lambda_{k}\right| \cos \frac{\pi s}{n+1}, \quad k=1,2 ; s=1,2, \cdots, n,
$$

where $\lambda_{1}$ and $\lambda_{2}$ are the eigenvalues of $R$.

There are three cases of interest.

(i) $R$ has real and distinct eigenvalues $\lambda_{1}, \lambda_{2}$.

(ii) $R$ has one real eigenvalue $\lambda$.

(iii) $R$ has complex eigenvalues $\lambda, \bar{\lambda}$.

The results of [14] were incomplete. We obtained a complete asymptotic answer in Case (i) and a partial answer in Case (iii). We did not discuss Case (ii). Actually one can obtain a complete asymptotic answer in Case (ii) by precisely the same methods as in Case (i). However, for completeness we shall]give a complete discussion of the problem.

Let 


$$
R=\left(\begin{array}{ll}
a & b \\
c & d
\end{array}\right)
$$

Then

(6.3a) $f(\theta)=\left[\begin{array}{cc}2 a \cos \theta & (b+c) \cos \theta+i(b-c) \sin \theta \\ (b+c) \cos \theta-i(b-c) \sin \theta & 2 d \cos \theta\end{array}\right]$,

and the eigenvalues of $f(\theta)$ are given by

$$
\rho(\theta)=(a+d) \cos \theta \pm D,
$$

where

$$
D=\left[D_{0} \cos ^{2} \theta+(b-c)^{2}\right]^{1 / 2},
$$

and

$$
D_{0}=(a-d)^{2}+4 b c .
$$

Let $\mu_{m}$ and $\mu_{M}$ be the minimal and maximal eigenvalues of $A_{n}=T_{n}[f]$ respectively. Then

$$
\left\|A_{n}\right\|=\max \left\{\left|\mu_{m}\right|,\left|\mu_{M}\right|\right\} \text {. }
$$

Since $\left\|A_{n}\right\|=\left\|-A_{n}\right\|$ we may assume

$$
(a+d) \geqq 0 \text {. }
$$

Since $-\lambda_{M}$ is the minimal eigenvalue of $-T_{n}[f]=T_{n}[-f]$ the results of the previous section lead us to study

$$
\mu(\theta)=(a+d) \cos \theta-D
$$

and

$$
\sigma(\theta)=-(a+d) \cos \theta-D,
$$

which are the minimal eigenvalue functions for $f(\theta)$ and $-f(\theta)$ respectively. Let us consider the separate cases.

Case (i). Let $\psi$ be the angle between the two eigenvectors of $R$. It was shown in [14] that

$$
b_{n-1} \leqq\left\|A_{n}\right\| \leqq b_{n+1}
$$

where

(6.6b) $\quad b_{k}=\left|\lambda_{1}+\lambda_{2}\right| \cos \frac{\pi}{k+1}+\left|\lambda_{1}-\lambda_{2}\right| \cdot\left[\cos ^{2} \frac{\pi}{k+1}+T^{2}\right]^{1 / 2}$, if

$$
T=\operatorname{ctn} \psi \text {. }
$$

Essentially this same result is obtained if we notice that 


$$
\begin{aligned}
\left|\lambda_{1}+\lambda_{2}\right| & =(a+d), \\
\left|\lambda_{1}-\lambda_{2}\right| & =\left(D_{0}\right)^{1 / 2}, \\
T^{2} & =\frac{(b-c)^{2}}{D_{0}},
\end{aligned}
$$

and hence

$$
\begin{aligned}
& \mu(\theta)=\left|\lambda_{1}+\lambda_{2}\right| \cos \theta-\left|\lambda_{1}-\lambda_{2}\right| \cdot\left\{\cos ^{2} \theta+T^{2}\right\}, \\
& \sigma(\theta)=-\left|\lambda_{1}+\lambda_{2}\right| \cos \theta-\left|\lambda_{1}-\lambda_{2}\right| \cdot\left\{\cos ^{2} \theta+T^{2}\right\} .
\end{aligned}
$$

Moreover $\mu(\theta+\pi)$ satisfies Conditions A and A(1) while $\sigma(\theta)$ satisfies Conditions $A$ and $A(1)$. Finally the associated eigenvector functions are even analytic in $\theta$. Thus, by Theorem 6

$$
\mu_{M}=-\mu_{m}=\left\|A_{n}\right\|=-\sigma\left(\frac{\pi}{n}\right)+o\left(\frac{1}{n}\right)^{2}
$$

Case (ii). If we apply the techniques of [14] to this case we obtain the following.

There is an orthogonal transformation $T$ such that

$$
T^{*} R T=T^{-1} R T=\left[\begin{array}{ll}
\lambda & 0 \\
\alpha & \lambda
\end{array}\right] .
$$

Then

$$
\beta_{n-1} \leqq\left\|A_{n}\right\| \leqq \beta_{n+1}
$$

where

$$
\beta_{k}=2|\lambda| \cos \frac{\pi}{k+1}+|\alpha| .
$$

If $\lambda \neq 0$ we obtain essentially the same result by noting that

$$
\begin{aligned}
2|\lambda| & =(a+d), \\
\alpha & =(b-c) .
\end{aligned}
$$

Thus we find

$$
\begin{aligned}
& \mu(\theta)=2|\lambda| \cos \theta-|\alpha|, \\
& \sigma(\theta)=-2|\lambda| \cos \theta-|\alpha| .
\end{aligned}
$$

As in Case (i), $\mu(\theta+\pi)$ and $\sigma(\theta)$ both satisfy Conditions A and A(1) and the eigenvectors are smooth. Hence, by Theorem 6

$$
\left\|A_{n}\right\|=2|\lambda| \cos \frac{\pi}{k+1}+|\alpha|+o\left(\frac{1}{n}\right)^{2} .
$$


If $\lambda=0$ our results on block Toeplitz matrices do not apply. However, (6.11) still holds. In fact, in virtue of (6.9c) we have

$$
\left\|A_{n}\right\| \equiv|\alpha| \quad \text { for all } n .
$$

Case (iii). Let $\lambda=s+i t$. Let $\xi, \bar{\xi}$ be the eigenvectors of $R$ associated with $\lambda$ and $\lambda$ respectively. Let $\eta$ and $\bar{\eta}$ be the corresponding eigenvectors of $R^{*}$. Let

$$
\tau=\left|\frac{(\bar{\xi}, \xi)}{(\bar{\xi}, \eta)}\right|,
$$

i.e. $\tau$ is the absolute value of ctn of the "angle" between $\xi$ and $\bar{\xi}$. If In [14] we obtained:

$$
\frac{s^{2}}{s^{2}+t^{2}}\left(1+\tau^{2}\right) \geqq 1
$$

then

$$
\left\|A_{n}\right\| \leqq 2|s| \cos \frac{\pi}{n+2}+2|t|\left[\sin ^{2} \frac{\pi}{n+2}+\tau^{2}\right]^{1 / 2} .
$$

We readily find that

$$
\begin{aligned}
2|s| & =(a+d) \\
2|t| & =\left(-D_{0}\right)^{1 / 2} \\
\tau^{2} & =-1-\frac{(b-c)^{2}}{D_{0}}
\end{aligned}
$$

and thus

$$
\begin{aligned}
& \mu(\theta)=2|s| \cos \theta-2|t|\left\{\sin ^{2} \theta+\tau^{2}\right\}^{1 / 2} \\
& \sigma(\theta)=-2|s| \cos \theta-2|t|\left\{\sin ^{2} \theta+\tau^{2}\right\}^{1 / 2} .
\end{aligned}
$$

However, it is not quite apparent how these functions behave. Proceeding in the obvious way, we find

$$
\begin{aligned}
& \dot{\mu}(\theta)=-2 \sin \theta\left\{|s|+\frac{|t| \cos \theta}{\left[\sin ^{2} \theta+\tau^{2}\right]^{1 / 2}}\right\}, \\
& \dot{\sigma}(\theta)=+2 \sin \theta\left\{|s|-\frac{|t| \cos \theta}{\left[\sin ^{2} \theta+\tau^{2}\right]^{1 / 2}}\right\} .
\end{aligned}
$$

We now see that there are three cases of interest.

Case a.

$$
\frac{s^{2}}{s^{2}+t^{2}}\left(1+\tau^{2}\right)>1
$$


In this case both $\mu(\theta+\pi)$ and $\sigma(\theta)$ satisfy Conditions $\mathrm{A}$ and $\mathrm{A}(1)$ and the corresponding eigenvectors are sufficiently smooth. Thus by Theorem 6

$$
\left\|A_{n}\right\|=-\sigma\left(\frac{\pi}{n}\right)+o\left(\frac{1}{n}\right)^{2}
$$

i.e.

$$
\left\|A_{n}\right\|=2|s| \cos \frac{\pi}{n}+2|t|\left\{\sin ^{2} \frac{\pi}{n}+\tau^{2}\right\}^{1 / 2}+o\left(\frac{1}{n}\right)^{2} .
$$

Case b.

$$
\frac{s^{2}}{s^{2}+t^{2}}\left(1+\tau^{2}\right)=1
$$

In this case both $\mu(\theta+\pi)$ and $\sigma(\theta)$ satisfy Conditions A and A(2). Again the eigenvectors are sufficiently smooth and Theorem 6 enables us to conclude

$$
\left\|A_{n}\right\|=-\sigma\left(\frac{4.73}{n}\right)+o\left(\frac{1}{n}\right)^{4},
$$

i.e.

(6.17) $\left\|A_{n}\right\|=2|s| \cos \frac{4.73}{n}+2|t| \cdot\left[\sin ^{2}\left(\frac{4.73}{n}\right)+\tau^{2}\right]^{1 / 2}+o\left(\frac{1}{n}\right)^{4} \cdot$

Case c.

$$
\frac{s^{2}}{s^{2}+t^{2}}\left(1+\tau^{2}\right)<1
$$

Let

$$
\theta_{0}=\cos ^{-1}\left[\frac{s^{2}}{s^{2}+t^{2}}\left(1+\tau^{2}\right)\right]^{1 / 2} .
$$

Then $\mu(\theta+\pi)$ and $\sigma(\theta)$ satisfy Conditions $\mathrm{B}$ and $\mathrm{B}(1)$ and the point $\theta_{0}$ is the point at which $\sigma(\theta)$ assumes its minimum.

This time we use Theorem 7 to conclude that:

$$
\left\|A_{n}\right\|=2|s| \cos \left(\theta_{0}+\frac{\pi}{n}\right)+2|t|\left\{\sin ^{2}\left(\theta_{0}+\frac{\pi}{n}\right)+\tau^{2}\right\}^{1 / 2}
$$

$$
+o\left(\frac{1}{n}\right)^{2} \text {. }
$$

Roughly, we have 


$$
\left\|A_{n}\right\|=2|\lambda|\left(1+\tau^{2}\right)^{1 / 2}-O\left(\frac{1}{n}\right)^{2} .
$$

We remark that if $R R^{*}=R^{*} R$ then $T^{2}, \alpha$ and $\tau^{2}$ are zero in Cases (i), (ii), and (iii) respectively and we obtain the same asymptotic results as given by (6.2).

7. The biharmonic difference equation. We will now apply the results of the preceding section and Theorem 8 to a problem in numerical analysis. In [14] we were concerned with the finite-difference equations which arise when we consider the following boundary-value problem for the biharmonic operator.

Let $D$ be the rectangular domain given by

$$
D: 0 \leqq x \leqq L, \quad 0 \leqq y \leqq M .
$$

Let $u=u(x, y)$ be the solution of the problem

$$
\Delta \Delta u=h(x, y) \text { in } D
$$

with $u$ and $u_{y}$ prescribed on the horizontal sides of $D$ while $u$ and $u_{x x}$ are prescribed on the vertical sides of $D$.

If we now write difference-equations to approximate (7.1b) (in the usual way) choosing

$$
\Delta_{x}=\frac{L}{p}, \quad \Delta_{y}=\frac{M}{q}
$$

we obtain a particular system of linear equations to solve.

It was shown in [14] that the "dominant" eigenvalue $\lambda_{R}$ of the horizontal two-line simultaneous-displacement (Richardson) iterative method may be determined as the spectral norm of a matrix of the form (6.1) where

$$
R=\left[\begin{array}{cc}
\frac{\alpha-c / 2}{b-c} & \frac{c}{2} \frac{1}{\left(b^{2}-c^{2}\right)^{1 / 2}} \\
-\frac{c}{2} \frac{1}{\left(b^{2}-c^{2}\right)^{1 / 2}} & \frac{\alpha-c / 2}{b+c}
\end{array}\right]
$$

where $n=2 /(q-1)$ and

$$
\begin{gathered}
b=\frac{1}{R}\left[L^{2}+2 \theta_{y}^{2}\right], \quad c=\frac{-2 \theta_{y}}{R} L, \\
L=1-2 \theta_{x} \cos \frac{\pi}{P}, \\
\theta_{x}=\frac{\Delta y^{2}}{2\left(\Delta x^{2}+\Delta y^{2}\right)}, \quad \theta_{y}=\frac{\Delta x^{2}}{2\left(\Delta x^{2}+\Delta y^{2}\right)} .
\end{gathered}
$$


Using the notation of $\S 6$ we have

$$
\rho(\theta)=\frac{2 \theta_{y}^{2}\left|L^{2}-\theta_{y}^{2}\right|}{L^{4}+\theta_{y}^{4}} \cos \theta \pm \frac{4 \theta_{y}^{8}|L|}{L^{4}+\theta_{y}^{4}} \cdot\left[\sin ^{2} \theta+\frac{L^{4}}{4 \theta_{y}^{4}}\right]^{1 / 2} .
$$

The eigenvalues of $R$ are complex,

$$
\lambda=\frac{\theta_{y}^{2}\left(2 \theta_{y}^{2}-L^{2}\right)}{L^{4}+4 \theta_{y}^{4}} \pm i \frac{2 \theta_{y}^{8} L}{L^{4}+4 \theta_{y}^{4}}
$$

and we must consider Case (iii) of $\$ 6$. Moreover, we find the interesting result that for any finite $p$ we are in Case a and for $p=\infty$ Case b applies.

Letting $t=1 / p, t_{0}=0, t_{1}=1 / 10$ we may apply Theorem 8 . There is no difficulty in verifying that the appropriate "smoothness" requirements are satisfied.

Let

$$
\lambda(x)=\frac{2 \theta_{y}^{2}\left|L^{2}-2 \theta_{y}^{2}\right|}{L^{4}+\theta_{y}^{4}} \cos x+\frac{4 \theta_{y}^{3}|L| \cdot\left[\sin ^{2} x+\frac{L^{4}}{4 \theta^{4}}\right]^{1 / 2}}{L_{y}^{4}+\theta_{y}^{4}} .
$$

We have

(i) for any finite $p$, as $q \rightarrow \infty$

$$
\lambda_{R}=\lambda\left(\frac{2 \pi}{q}+\circ\left(\frac{1}{q}\right)\right)
$$

and

(ii) for all $p$ and $p, q \rightarrow \infty$

$$
\lambda\left(\frac{9.46}{q}+o\left(\frac{1}{q}\right)\right) \leqq \lambda_{R} \leqq \lambda\left(\frac{2 \pi}{q}+o\left(\frac{1}{q}\right)\right) .
$$

We may write (7.7b) as follows. Let

$$
\psi=\frac{1}{2}\left(\frac{\theta_{x}}{\theta_{y}}\right)^{2}\left(\frac{\pi}{p}\right)^{4}+\frac{1}{4}\left(\frac{\theta_{x}}{\theta_{y}}\right)\left(\frac{\pi}{p}\right)^{2}\left(\frac{9.46}{q}\right)^{2}+\frac{1}{32}\left(\frac{9.46}{q}\right)^{4}
$$

and

$$
\Theta=\left(\frac{\theta_{x}}{p^{2}}+\frac{\theta_{y}}{q^{2}}\right)
$$

Then

(7.9) $1-\psi+o\left(\frac{1}{p^{2}} \frac{1}{q^{2}}+\frac{1}{q^{5}}\right) \leqq \lambda_{R} \leqq 1-\frac{\pi^{4}}{2 \theta_{v}^{2}} \Theta^{2}+o\left(\frac{1}{p^{2} q^{3}}+\frac{1}{q^{5}}\right)$. 
In practice $p / q=O(1)$ and we may write

$$
1-\psi \lesssim \lambda_{R} \lesssim 1-\frac{\pi^{4}}{2 \theta_{\nu}^{2}} \Theta^{2} .
$$

8. Elliptic difference equations. Consider the finite-difference equations which arise in connection with the following boundary-value problem.

Let $D$ be the rectangular domain given by

$$
D: 0 \leqq x \leqq L, \quad 0 \leqq y \leqq M .
$$

Let $u=u(x, y)$ be the solution of the problem

$$
A U_{x x}+2 B U_{x y}+C U_{y y}=h(x, y) \text { in } D,
$$

with $u$ prescribed on the boundary of $D$ and $A, B$, and $C$ are constants satisfying

$$
A>0, \quad A C-B^{2}>0 .
$$

Choosing integers $p$ and $q$ and setting

$$
\Delta x=\frac{L}{p}, \quad \Delta y=\frac{M}{q},
$$

the "natural" finite-difference equations are

$$
\begin{aligned}
& v_{r s}-a\left(v_{r+1,8}+v_{r-1, \ell}\right)-c\left(v_{r, \ell+1}+v_{r, \&-1}\right) \\
& -\frac{1}{2} b\left(v_{r+1, \ell+1}+v_{r-1, \ell-1}-v_{r-1, \&+1}-v_{r+1, \ell-1}\right) \\
& \quad=y_{r s}, 1 \leqq r \leqq p-1,1 \leqq s \leqq q-1,
\end{aligned}
$$

where $v_{0, e}, v_{p, s}, v_{r, 0}$, and $v_{r, q}$ are the given boundary values and

$$
a=\frac{A(\Delta y)^{2}}{D}, \quad c=\frac{C(\Delta x)^{2}}{D}, \quad b=\frac{B \Delta x \Delta y}{D},
$$

$$
\begin{aligned}
y_{r, e} & =-\frac{h_{r s}(\Delta x)^{2}(\Delta y)^{2}}{D}, \\
D & =2\left[A(\Delta y)^{2}+C(\Delta x)^{2}\right] .
\end{aligned}
$$

Hence

$$
a+c=\frac{1}{2}, \quad a c-b^{2}>0
$$

The matrix representation of (8.2) may be written as

$$
M X=Y
$$


where $M$ is a block Toeplitz matrix. In fact this may be done in two ways.

Horizontal representation. We choose to number the unknowns as follows:

$$
v_{r s}=X_{(p-1)(r-1)+\varepsilon-1}
$$

i.e. we count from left to right along horizontal lines starting at the bottom. Then (8.2) may be written in the form

$$
T_{q-2}[f] X=Y
$$

where $f(\theta)$ is a Hermitian matrix of order $p-1$ of the form

$$
f(\theta)=L+A e^{i \theta}+A^{*} e^{-i \theta}
$$

with

$$
\begin{aligned}
& L=I_{p-1}-a\left(J_{p-1}+J_{p-1}^{*}\right) \\
& A=-c I_{p-1}+\frac{1}{2} b\left(J_{p-1}-J_{p-1}^{*}\right)
\end{aligned}
$$

where $J_{p-1}=\left(\delta_{i, j-1}\right)$ as in $\S 5$.

Vertical representation. We choose to number the unknowns as follows:

$$
V_{r, s}=\xi_{(r-1)+(q-1)(8-1)}
$$

i.e., we count along vertical lines.

In this representation (8.2) may be written in the form

$$
T_{p-2}[h] \xi=\eta,
$$

where $h(\psi)$ is a Hermitian matrix of order $q-1$ of the form

$$
h(\psi)=R+B e^{i \psi}+B^{*} e^{-1 \psi}
$$

with $R=I_{q-1}-c\left(J_{q-1}+J_{q-1}^{*}\right)$,

$$
B=-a I_{q-1}-\frac{1}{2} b\left(J_{q-1}-J_{q-1}^{*}\right) .
$$

Another interesting fact is this: both $f(\theta)$ and $h(\theta)$ are themselves scalar Toeplitz matrices for every $\theta$ !!

The fact that (8.2) has two block Toeplitz representations and both functions are themselves Toeplitz matrices comes about because the operator involved is a two-dimensional Toeplitz matrix. Such matrices will be discussed more generally in $\$ 9$.

We now turn to the problem of solving those equations and consider two iterative methods.

(1.) "Point" Richardson (simultaneous displacement). Choosing a "guess" $X^{0}$ we iterate as follows: 


$$
X^{(n+1)}=-\left(T_{q-2}[f]-I\right) X^{(n)}+Y .
$$

This method converges if and only if

$$
\left\|T_{q-2}[f]-I\right\|<1
$$

and

$$
\left\|T_{q-2}[f]-I\right\|=\max |\lambda|
$$

where $\lambda$ is an eigenvalue of $T_{q-2}[f]-I=T_{q-2}[f-I]$.

In anticipation of applying the results of $\$ 5$ we determine the eigenvalues of $f(\theta)-I$. This is easily done, indeed all the eigenvalues of $f(\theta)-I$ are given by

$$
\rho_{s}(\theta)=2 c \cos \theta+2\left\{a^{2}+b^{2} \sin ^{2} \theta\right\}^{1 / 2} \cos \frac{\pi s}{p}, \quad s=1,2, \cdots, p-1 .
$$

Thus we must consider

$$
\mu(\theta)=2 c \cos \theta-2\left\{a^{2}+b^{2} \sin ^{2} \theta\right\}^{1 / 2} \cos \frac{\pi}{p}
$$

and

$$
\sigma(\theta)=-\left\{2 c \cos \theta+2\left\{a^{2}+b^{2} \sin \theta\right\}^{1 / 2} \cos \frac{\pi}{p}\right\},
$$

which are the minimal eigenvalues of $f(\theta)-I$ and $I-f(\theta)$ respectively.

Using (8.2b) it is not difficult to determine that both $\mu(\theta)$ and $\sigma(\theta)$ satisfy Conditions $\mathrm{A}$ and $\mathrm{A}(1)$. Thus, using the argument of Theorem 6,

$$
\lambda_{R} \leqq 2 c \cdot \cos \frac{\pi}{q}+2 a \cdot \cos \frac{\pi}{p}+\frac{b^{2}}{a} \sin ^{2} \frac{\pi}{q} \cos \frac{\pi}{p}+o\left(\frac{1}{p^{2}}+\frac{1}{q^{2}}\right)
$$

for all sufficiently large $p$ and $q$. Moreover since the eigenvalues are all distinct it is easy to verify that the corresponding eigenvectors are analytic in $\theta$. Hence we may apply Theorem 6. Hence we may conclude: for fixed $p$

$$
\begin{aligned}
& \left\|T_{q-2}[f]-I\right\| \\
& \quad=2 c \cos \frac{\pi}{q}+2\left\{a^{2}+b^{2} \sin ^{2} \frac{\pi}{q}\right\}^{1 / 2} \cos \frac{\pi}{p}+o\left[\left(\frac{1}{q}\right)^{2}\right] .
\end{aligned}
$$

To get a more complete answer to our question we note that the eigenvalues of $T_{p-2}[h]-I$ are the same as those of $T_{q-2}[f]-I$.

The eigenvalues of $h-I$ are found to be

$$
\tau_{\bullet}(\theta)=2 a \cdot \cos \theta+2\left\{c^{2}+b^{2} \sin ^{2} \theta\right\}^{1 / 2} \cos \frac{\pi s}{q}, \quad s=1,2, \cdots, q-1 .
$$


And in exactly the same way as before we conclude:

(8.9a) $\quad \lambda_{R} \leqq 2 c \cdot \cos \frac{\pi}{q}+2 a \cdot \cos \frac{\pi}{p}+\frac{b^{2}}{c} \sin ^{2} \frac{\pi}{p} \cos \frac{\pi}{q}+o\left(\frac{1}{p^{2}}+\frac{1}{q^{2}}\right)$

for all sufficiently large $p$ and $q$. Moreover for fixed $q$

$$
\left\|T_{p-2}[h]-I\right\|=2 a \cdot \cos \frac{\pi}{p}+2\left\{c^{2}+b^{2} \sin ^{2} \frac{\pi}{p}\right\}^{1 / 2} \cos \frac{\pi}{q}+o\left(\frac{1}{p}\right)^{2} .
$$

Hence, for all $p$ and $q$

$$
\begin{aligned}
\max |\lambda| \leqq & 1-\left[c\left(\frac{\pi}{q}\right)^{2}+a\left(\frac{\pi}{p}\right)^{2}\right]+\min \left\{\frac{b^{2}}{c}\left(\frac{\pi}{p}\right)^{2}, \frac{b^{2}}{a}\left(\frac{\pi}{q}\right)^{2}\right\} \\
& +o\left(\left(\frac{1}{p}\right)^{2}+\left(\frac{1}{q}\right)^{2}\right) .
\end{aligned}
$$

We now consider another iterative approach.

(2.) "Horizontal line" Richardson. Let

$$
f(\theta)-L=M(\theta) .
$$

Again, choosing an initial "guess" $X^{0}$ we iterate as follows:

$$
T_{q-2}[L] X^{(n+1)}=-T_{q-2}[M] X^{(n)}+Y .
$$

It is useful to observe that if

$$
P(\psi)=(1-2 a \cdot \cos \psi) I_{q-1}
$$

and

$$
-N(\psi)=h(\psi)-P(\psi),
$$

then using the vertical representation we may write $(8.13 \mathrm{a})$ as

$$
T_{p-2}[P] \xi^{(n+1)}=T_{p-2}[N] \xi^{(n)}+\eta .
$$

In this case, the iterative procedure converges (see [4]) if and only if

$$
\max |\lambda|<1
$$

where $\lambda$ is a solution of the equation

$$
\operatorname{det}\left(T_{q-2}[\lambda L+M]\right)=0
$$

or $\lambda$ (equivalently) is a solution of

$$
\operatorname{det}\left(T_{p-2}[\lambda P-N]\right)=0 .
$$

Since this iterative method is a "three block scheme" (see [4]) it is not 
difficult to show that if $\lambda$ is a root of (8.15b) so is $-\lambda$. Hence we restrict ourselves to positive $\lambda$.

Our approach is now the following. Assuming $\lambda>0$ we obtain the minimal eigenvalue of $T_{q-2}[\lambda L+M]$ (or $T_{p-2}[\lambda P-N]$ ) as a function of $\lambda$. We then set that minimal eigenvalue equal to zero and solve for $\lambda$. This value is the desired $\max |\lambda|$ since the positive-definite character of $L$ shows that for $\lambda>0$, $\operatorname{det}\left(T_{q-2}[\lambda L+M]\right)$ is monotone increasing.

As before, we turn to eigenvalues of $\lambda L+M$ and $\lambda P-N$. All the eigenvalues of $\lambda P-N$ are given by

$$
\begin{aligned}
\rho_{s}(\theta)=\lambda(1-2 a \cdot \cos \theta)-2\left\{c^{2}+b^{2} \sin ^{2} \theta\right\}^{1 / 2} \cos \frac{\pi s}{q} & \\
& \\
s & =1,2, \cdots, q-1 .
\end{aligned}
$$

Once more we find distinct eigenvalues and the smoothness of the eigenvector functions is assured. The minimal eigenvalue is

$$
\mu(\theta)=\lambda(1-2 a \cdot \cos \theta)-2\left\{c^{2}+b^{2} \sin ^{2} \theta\right\}^{1 / 2} \cos \frac{\pi}{q}
$$

which satisfies Conditions $\mathrm{A}$ and $\mathrm{A}(1)$. Theorem 6 allows us to conclude that the minimal eigenvalue $\sigma$ of $T_{p-2}[\lambda P-N]$ is

$$
\sigma=\lambda\left(1-2 a \cdot \cos \frac{\pi}{p}\right)-2\left\{c^{2}+b^{2} \sin ^{2} \frac{\pi}{p}\right\}^{1 / 2} \cos \frac{\pi}{q}+o\left(\frac{1}{p}\right)^{2}
$$

Thus we may conclude: for fixed $q$

$$
\lambda_{R}=\frac{2\left\{c^{2}+b^{2} \sin ^{2}(\pi / p)\right\}^{1 / 2} \cos (\pi / q)}{1-2 a \cos (\pi / p)}+o\left[\left(\frac{1}{p}\right)^{2}\right] .
$$

It is important to notice that (8.18) implies

$$
\lambda_{R}=1-O\left(\frac{1}{p^{2}}+\frac{1}{q^{2}}\right) \text {. }
$$

All the eigenvalues of $\lambda L+M$ are also easily found. We obtain

$$
\begin{aligned}
\sigma_{s}(\theta)=\lambda-2 c \cos \theta-2\left\{\lambda^{2} a^{2}+b^{2} \sin ^{2} \theta\right\}^{1 / 2} \cos \frac{\pi s}{p} & \\
& s=1,2, \cdots, p-1 .
\end{aligned}
$$

Once more eigenvalues are distinct and the minimal eigenvalue $\mu(\theta)$ satisfies Conditions $\mathrm{A}$ and $\mathrm{A}(1)$. Thus we may apply Theorem 6 and obtain the minimal eigenvalue of $T_{q-2}[\lambda L+M]$. Using (8.19) we have 


$$
\begin{aligned}
\sigma= & \lambda\left[1-2\left(a^{2}-b^{2}\left(1+o\left(\frac{1}{p}\right)^{2}\left(\sin ^{2} \frac{\pi}{q}\right)^{1 / 2} \cos \frac{\pi}{p}\right]\right.\right. \\
& -2 c \cos \frac{\pi}{q}+o\left(\frac{1}{q}\right)^{2} .
\end{aligned}
$$

Thus we may conclude: for fixed $p$

$$
\lambda_{R}=\frac{2 c \cos (\pi / q)}{1-2\left[a^{2}+b^{2}\left(1+O(1 / p)^{2}\right) \sin ^{2}(\pi / q)\right]^{1 / 2} \cos (\pi / p)}+o\left(\frac{1}{q}\right)^{2} .
$$

Finally, we have, just as in the previous case, as $p$ and $q \rightarrow \infty$

$$
\begin{aligned}
\lambda_{R} \leqq & 1-\frac{1}{2 c}\left[c\left(\frac{\pi}{q}\right)^{2}+a\left(\frac{\pi}{p}\right)^{2}\right]+\min \left\{\frac{b^{2}}{2 c^{2}}\left(\frac{\pi}{p}\right)^{2}, \frac{b^{2}}{2 a c}\left(\frac{\pi}{q}\right)^{2}\right\} \\
& +o\left[\left(\frac{1}{p}+\frac{1}{q}\right)^{2}\right] .
\end{aligned}
$$

Remarks. Since the "line" iterative methods are "three block schemes" (8.22) enables one to estimate the principle eigenvalues of several other iterative schemes. See $[4 ; 14$ or 11$]$ for more details. If $B=0$ we are in a wellstudied case, and our results give the well-known results for that case. See [14] for more details.

It should be remarked that the estimates (8.10) and (8.22) are not really new results. It is easy to see that in fact, for the point iteration scheme we have

$$
\lambda_{R} \approx 1-\frac{\lambda \cdot \Delta x^{2} \cdot \Delta y^{2}}{D}
$$

where $\lambda$ is the minimal eigenvalue of the equation

$$
A U_{x x}+2 B U_{x y}+C U_{y y}+\lambda U=0
$$

with $U=0$ on the boundary of the rectangle. By a change of variables $\lambda$ is seen to be the minimal eigenvalue of

$$
\Delta U+\lambda U=0
$$

with $U=0$ on the boundary of a particular parallelogram. The estimates (8.7a) and (8.9a) are easily related to the lower estimates obtained for $\lambda$ by Steiner Symmetrization. (See Pólya and Szegö [22].)

9. Remarks on two-dimensional Toeplitz matrices. Let $f(\theta, \psi)$ be a real Lebesgue integrable function defined for $-\pi \leqq \theta, \psi \leqq \pi$. Let $\left\{C_{j k}\right\}$ be the Fourier coefficients of $F(\theta, \psi)$, i.e.

$$
F(\theta, \psi) \sim \sum_{k, j} C_{j k} e^{i j \theta} e^{i k \psi}
$$


One may now regard $F(\theta, \psi)$ as a function of $\theta$ depending on a parameter $\psi$ or as a function of $\psi$ depending on a parameter $\theta$. To distinguish these cases we define

$$
\begin{aligned}
& A(\theta)=F(\theta, \psi) \text { for fixed } \psi, \\
& B(\psi)=F(\theta, \psi) \text { for fixed } \theta .
\end{aligned}
$$

That is,

$$
\begin{aligned}
& A(\theta) \sim \sum_{j} \gamma_{j}(\psi) e^{i j \theta}, \\
& B(\psi) \sim \sum_{k} \delta_{k}(\theta) e^{i k \theta}
\end{aligned}
$$

where

$$
\begin{aligned}
\gamma_{j} & \sim \sum_{k} C_{j k} e^{i k \psi} . \\
\delta_{k} & \sim \sum_{j} C_{j k} e^{i j 6}
\end{aligned}
$$

We observe that

$$
T_{n}[A(\theta)]=h(\psi)
$$

and

$$
T_{N}[B(\psi)]=f(\theta)
$$

are both matrix valued functions which are Hermitian and periodic with period $2 \pi$. Moreover, both are scalar Toeplitz matrices for all values of their arguments.

Consider now the two block Toeplitz matrices of order $(n+1)(N+1)$

$$
m=T_{N}[h]=T_{N}\left[T_{n}(A)\right]
$$

and

$$
M=T_{n}[f]=T_{n}\left[T_{N}(B)\right] .
$$

Let $X$ be any vector of dimension $(n+1)(N+1)$. Let $X=\left(X_{k}\right), k=0,1,2$, $\cdots, N+1$ where the $X_{k}$ are themselves $(n+1)$ vectors, say

$$
X_{k}=\left(\xi_{j}^{(k)}\right),
$$$$
j=0,1, \cdots, n .
$$

Let $G(\psi)=\sum_{k=0}^{n} X_{k} e^{-i k \psi}$ and

$$
h(\theta, \psi)=\sum_{j=0}^{N} \sum_{k=0}^{n} \xi_{j}^{(k)} e^{-i k \psi} e^{-i j \theta .}
$$

Then 
(9.4c) $\quad(X, m X)=\frac{1}{2 \pi} \int_{-\pi}^{\pi} G^{*} h G d \psi=\frac{1}{4 \pi^{2}} \int_{-\pi}^{\pi} \int_{-\pi}^{\pi} F(\theta, \psi)|h|^{2} d \theta d \psi$.

A similar statement holds for the matrix $M$.

It is not difficult to verify the following statements.

(S.1) There exists a permutation matrix $P$ so that

$$
P^{*} m P=P^{-1} m P=M .
$$

(S.2) If $\lambda_{1, n}$ is the minimum eigenvalue of $m$ and $M$, then

$$
\lambda_{1, n}=\min \frac{1}{4 \pi^{2}} \int_{-\pi}^{\pi} \int_{-\pi}^{\pi} F(\theta, \psi)|h(\theta, \psi)|^{2} d \theta d \psi
$$

where $h(\theta, \psi)$ is any function of the form (9.4b) and

$$
\frac{1}{4 \pi^{2}} \int_{-\pi}^{\pi} \int_{-\pi}^{\pi}|h(\theta, \psi)|^{2} d \theta d \psi=1
$$

(S.3) The matrices $m$ and $M$ correspond to two-dimensional difference equations in the same sense that the one-dimensional (scalar) Toeplitz matrices correspond to ordinary difference equations. That is, if $F(\theta, \psi)$ is a trigonometric polynomial the equations

$$
m X=Y
$$

represent a partial difference equation in two variables of the form

$$
\sum_{k=-0}^{\sigma} \sum_{j=-\infty}^{\rho} C_{j k} v_{r+k, \ell+j}=y_{r, e}
$$

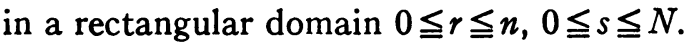

Conversely, any difference equation of the form (9.6) may be represented by a matrix $m$ or a matrix $M$ which are related in this way and $F(\theta, \psi)$ will be a trigonometric polynomial.

REMARK. If one is dealing with a time-dependent difference equation and $v_{r, 8}$ represents the unknown function at time $\nu \Delta t$ while $y_{r, s}$ represents that function at time $(\nu+1) \Delta t$, the function $F(\theta, \psi)$ is the "amplification" in the von Neumann theory of stability. See [15].

As an illustration of these facts one can turn to the difference equation (8.2). In this case

$$
F(\theta, \psi)=1-2 c \cdot \cos \theta-2 a \cos \psi+b \sin \theta \sin \psi
$$

At this time there is no general theory of such two-dimensional Toeplitz operators. However, the results of $\S 8$ show that one can apply the preceding theory to obtain some useful estimates. 


\section{REFERENCES}

1. R. J. Arms, L. D. Gates and B. Zondek, A method of block iteration, J. Soc. Indust. Appl. Math. vol. 4 (1956) pp. 220-229.

2. S. D. Conte and R. T. Dames, An alternating direction scheme for the biharmonic difference equations, Math. Tables Aids Comput. vol. 12 (1958) pp. 198-205.

3. R. Courant and D. Hilbert, Methods of mathematical physics, Vol. I. (English translation) New York, Interscience, 1953.

4. B. Friedman, The iterative solution of elliptic difference equations, A.E.C. Research and Development Report NYO-7698, New York University, 1957.

5. Stanley P. Frankel, Convergence rates of iterative treatments of partial differential equations, Math. Tables Aids Comput. vol. 4 (1950) pp. 65-76.

6. U. Grenander and G. Szegö, Toeplitz forms and their applications, Berkeley, University of California Press, 1958.

7. J. Heller, Simultaneous, successive and alternating direction iteration schemes, J. Soc. Indust. Appl. Math. vol. 8 (1960) pp. 150-173.

8. F. B. Hildebrand, Introduction to numerical analysis, New York, McGraw-Hill, 1956.

9. E. Jahnke and F. Emde, Tables of functions with formulae and curves, New York, Dover Publications, 1943.

10. M. Kac, W. L. Murdoch, and G. Szegö, On the eigenvalues of certain Hermitian forms, J. Rational Mech. Anal. vol. 2 (1953) pp. 767-800.

11. H. Keller, On some iterative methods for solving elliptic difference equations, Quart. Appl. Math. vol. 16 (1958) pp. 209-226.

12. C. C. MacDuffee, The theory of matrices. Ergebnisse der Mathematik und ihrer Grenzgebiete, vol. 2, Berlin, Springer, 1933.

13. L. M. Milne-Thomson, The calculus of finite-differences, New York, Macmillan, 1933.

14. S. V. Parter, On "two-line" iterative methods for the Laplace and biharmonic difference equations, Numer. Math. vol. 1 (1959) pp. 240-252. 1958.

15. R. D. Richtmyer, Difference methods for initial-value problems, New York, Interscience,

16. D. E. Rutherford, Some continuant determinants arising in physics and chemistry. II, Proc. Roy. Soc. Edinburgh Sect. A. vol. 63 (1952) pp. 232-241.

17. Richard S. Varga, Iterative numerical analysis, Lecture Notes, Computation and Data Processing Center, University of Pittsburgh, 1959.

18. - Factorization and normalized iterative methods, Presented at the Symposium on Boundary Problems in Differential Equations at University of Wisconsin, April 1959, Westinghouse Report W.A.P.D.-T. 1950.

19. H. Widom, On the eigenvalues of certain Hermitian operators, Trans. Amer. Math. Soc. vol. 88 (1958) pp. 491-522.

20. D. Young, Iterative methods for solving partial difference equations of elliptic type, Trans. Amer. Math. Soc. vol. 76 (1954) pp. 92-111.

21. A. Zygmund, Trigonometrical series, New York, Dover, 1955.

22. G. Polya and G. Szegö, Isoperimetric inequalities in mathematical physics, Annals of Mathematics Studies, No. 27, Princeton University Press, 1951.

CORNELl University, ITHACA, NEW YORK.

BROOKHAVEN NATIONAL LABORATORY, UPTON, LONG IsLAND, NEw YORK 